Pacific

Journal of

Mathematics

ROLLING CONSTRUCTION FOR ANISOTROPIC DELAUNAY SURFACES

Miyuki Koiso And Bennett Palmer

Volume $234 \quad$ No. 2

February 2008 


\title{
ROLLING CONSTRUCTION FOR ANISOTROPIC DELAUNAY SURFACES
}

\author{
Miyuki Koiso And Bennett Palmer
}

\begin{abstract}
Anisotropic Delaunay surfaces are surfaces of revolution that have constant anisotropic mean curvature. We show how the generating curves of such surfaces can be obtained as the trace of a point held in a fixed position relative to a curve that is rolled without slipping along a line. This generalizes the Delaunay's classical construction for surfaces of revolution with constant mean curvature. Our result is given as a corollary of a new geometric description of the rolling curve of a general plane curve. Also, we characterize anisotropic Delaunay curves by using their isothermic self-duality.
\end{abstract}

\section{Introduction}

When immiscible materials come into contact, the interface that forms between them is often modeled as a surface. According to the law of least action, the equilibrium surface will form so as to attempt to minimize its free potential energy subject to whatever constraints and additional forces are imposed by its environment.

In a liquid/air interface with no additional forces present, the free energy assigned to the interface is its surface tension. For homogeneous materials, the surface tension is proportional to the area of the surface interface. Minimization leads to the formation of what are known as minimal surfaces (when no volume constraints are imposed) and constant mean curvature surfaces (when a volume constraint is imposed).

If the temperature is gradually lowered, the liquid in the drop may crystallize. This means that its constituent molecules will be found in an ordered configuration and, as a result, the energy assigned to the surface interface will become anisotropic; it will depend on the direction of the surface at each point. The simplest formulation of an anisotropic surface energy is this: Let $F$ be a smooth

MSC2000: primary 58E12; secondary 49Q10.

Keywords: roulette, rolling curve, anisotropic, Delaunay surface.

Koiso is partially supported by Grant-in-Aid for Scientific Research (C) No. 16540195 of the Japan Society for the Promotion of Science. 
positive function defined on the two dimensional sphere $S^{2}$. If $v$ is a choice of a unit surface normal along the surface $\Sigma$, then

$$
\mathscr{F}:=\int_{\Sigma} F(v) d \Sigma
$$

defines an anisotropic surface energy called a constant coefficient parametric functional.

The surfaces that we will discuss here are in equilibrium among surfaces with prescribed volume for such parametric functionals which in addition satisfy an ellipticity condition: Denote by $D F$ and $D^{2} F$ the gradient and Hessian of $F$ on $S^{2}$. Then we require that at each point in $S^{2}$ the matrix $D^{2} F+F 1$ is positive definite. The functional appearing in (1-1) is referred to as a (constant coefficient) parametric elliptic functional. This means that the Euler-Lagrange equation which characterizes equilibria is an elliptic equation. The major consequence of ellipticity is a maximum principle analogous to that for constant mean curvature surfaces. Moreover, for such a parametric elliptic functional $\mathscr{F}$, there is a unique minimizer (up to translation in $\mathbb{R}^{3}$ ) of $\mathscr{F}$ among closed surfaces bounding a prescribed volume, which is called the Wulff shape (for $\mathscr{F}$ ), and it is a smooth convex surface.

When considering a nonlinear theory, it is essential to have a class of tractable examples at hand. When the energy functional is rotationally invariant (that is, the corresponding Wulff shape is a surface of revolution), an obvious class of examples to consider are the surfaces of revolution. In the classical case of isotropic surface energy, the surfaces of revolution with constant mean curvature were found by $\mathrm{C}$. Delaunay [1841]. The Delaunay surfaces are divided into major types: spheres, unduloids, catenoids, nodoids, and cylinders. Delaunay found an ingenious method for describing the generating curves of these surfaces. If a conic section $\Gamma$ rolls without slipping along a line, then the trace of one of its foci defines a curve $\Omega$ which, when rotated around the line, gives a constant mean curvature surface $\Sigma$. In particular, when $\Gamma$ is a parabola, $\Sigma$ is a catenoid; when $\Gamma$ is an ellipse, $\Sigma$ is an unduloid; and when $\Gamma$ is a hyperbola, we obtain a nodoid. Among the reasons for the importance of the Delaunay surfaces are these:

(1) They are the most accessible examples of constant mean curvature surfaces.

(2) In many problems involving symmetry, one can conclude a priori that the solution is a Delaunay surface.

(3) Because of (1), they serve as ideal comparison surfaces in applying the maximum principle to study other surfaces of prescribed mean curvature.

(4) The ends of any properly embedded surface with nonzero constant mean curvature in Euclidean space are asymptotic to Delaunay surfaces. 
(5) They are the essential building blocks in the construction of other important examples of constant mean curvature surfaces using "gluing methods."

It is reasonable to believe that the rotationally invariant equilibrium surfaces should play an analogously important role for other functionals besides surface area. In view of (2) and (3) above, this should particularly be the case for functionals having a maximum principle, as do the elliptic parametric functionals. In fact, in [Koiso and Palmer 2006; 2007a; 2007b] the maximum principle has been applied to conclude that solutions of capillary problems for rotationally invariant elliptic functionals with free boundaries on horizontal planes are surfaces of revolution.

This paper is organized as follows. Section 2 rederives the basic equations defining anisotropic Delaunay surfaces. We also give a generalization to the case where the Wulff shape need not be rotationally symmetric but is a product of convex curves. Section 3 reviews a general rolling construction of Hsiang and Yu and relates it to the classical concept of isothermic duality. We also give a refinement of the general rolling construction by determining the half-plane that contains the rolling curve (Theorem 3.1). In Section 4, we give the definition and some basic properties of the mean curvature profile associated with a surface $S$ of revolution. This is a curve whose curvature $\kappa(s)$ is equal to twice the mean curvature $H(s)$ measured along a meridian of $S$. Section 5 applies the concept of the mean curvature profile of a surface of revolution $S$ to give a geometric description of the rolling curve of the generating curve of $S$. The rolling curve $\Gamma$ is obtained as a type of dual curve of the mean curvature profile (Theorem 5.1). We then proceed to apply the general rolling construction to the anisotropic Delaunay surfaces, beginning with the Wulff shape in Section 6 (Theorem 6.1) and continuing with the other types of anisotropic Delaunay surfaces in Section 7 (Theorems 7.1, 7.2). In the applications to anisotropic unduloids and nodoids, the isothermic self-duality of their generating curves (Lemmas 7.4, 7.5) is used essentially. And in Section 8, we use isothermic duality to characterize the periodic curves that arise as generating curves of anisotropic unduloids and nodoids (Theorem 8.1).

\section{Generalized anisotropic Delaunay surfaces}

Here we will give a description of some equilibrium surfaces for functionals whose Wulff shape may not be rotationally symmetric. The Wulff shape is assumed to have the property that all of its intersections with horizontal planes are mutually homothetic. In the case when the Wulff shape is a surface of revolution, the construction reduces to that of the anisotropic Delaunay surfaces which were extensively studied in [Koiso and Palmer 2005]; that derivation was less elementary than the one appearing here. 
Let

$$
\Omega_{W}:(u(\sigma), v(\sigma)) \text { for }-2 L_{1} \leq \sigma \leq 2 L_{2}
$$

be a closed convex curve in the two-dimensional Euclidean space $\mathbb{R}^{2}$ parametrized by arc length $\sigma$. Here we mean by "a closed convex curve" a closed $C^{\infty}$-curve with positive curvature with respect to the inward pointing normal. We assume that $\Omega_{W}$ is symmetric with respect to the $v$-axis. We may assume that

$$
\begin{array}{lll}
u \geq 0 \quad \text { and } \quad v_{\sigma} \geq 0 & \text { for }-L_{1} \leq \sigma \leq L_{2}, \\
u<0 & \text { and } \quad v_{\sigma}<0 & \text { for } \sigma \in\left[-2 L_{1},-L_{1}\right) \cup\left(L_{2}, 2 L_{2}\right]
\end{array}
$$

Also we may assume that $u$ and $v^{\prime}$ have zeros only at $\sigma=-L_{1}, L_{2}$ and that $u^{\prime}$ and $v$ have zeros only at $\sigma=0,-2 L_{1}, 2 L_{2}$. Let $C: \tau \rightarrow(\alpha(\tau), \beta(\tau))$ be a closed convex curve parametrized by arc length in the plane. We assume that the origin is inside the domain bounded by $C$. Consider the surface $W$ in $\mathbb{R}^{3}$ given by

$$
\chi(\sigma, \tau)=(u(\sigma) \alpha(\tau), u(\sigma) \beta(\tau), v(\sigma)) .
$$

When $(\alpha, \beta)=(\cos \tau, \sin \tau)$, this gives a surface of revolution.

In general, $W$ is a convex surface such that all the curves obtained by intersecting $W$ with horizontal planes (that is, the third component $=$ constant) are homothetic to each other.

For a suitable plane curve $(x(s), z(s))$ parametrized by arc length with $x>0$, we have the surface $\Sigma$ defined by

$$
X(s, \tau)=(x(s) \alpha(\tau), x(s) \beta(\tau), z(s)) .
$$

A straightforward calculation shows that

$$
\chi_{\sigma} \times \chi_{\tau}=u\left(-v_{\sigma} \beta_{\tau}, v_{\sigma} \alpha_{\tau}, u_{\sigma}\left(\alpha \beta_{\tau}-\beta \alpha_{\tau}\right)\right) .
$$

Note that because of the assumption that $C=(\alpha, \beta)$ is convex, and because the origin $(0,0)$ is inside of $C$, the difference $\alpha \beta_{\tau}-\beta \alpha_{\tau}$ has a definite sign, and we can choose the orientation of this curve so that the sign is positive.

The same calculation holds for $X$ and so

$$
X_{s} \times X_{\tau}=x\left(-z_{s} \beta_{\tau}, z_{s} \alpha_{\tau}, x_{s}\left(\alpha \beta_{\tau}-\beta \alpha_{\tau}\right)\right) .
$$

Formulas (2-4) and (2-5) show that the normals to the two surfaces agree exactly when either

$$
\begin{array}{lll}
x_{s}=u_{\sigma} & \text { and } & z_{s}=v_{\sigma}, \\
x_{s}=-u_{\sigma} & \text { and } & z_{s}=-v_{\sigma} .
\end{array}
$$


Recall that the Gauss map of a convex surface is a diffeomorphism onto the 2sphere. The assignment to a point $X(s, \tau)$ on $\Sigma$ the point $\chi(\sigma(s), \tau)$ on $W$ where either in (2-6) holds defines a map which we call the anisotropic Gauss map of $X$. This map clearly factors through the 2 -sphere. We denote this map by $\underline{\chi}$.

We have

$$
\begin{aligned}
& \underline{\chi}_{s}=\sigma_{s}\left(u_{\sigma} \alpha, u_{\sigma} \beta, v_{\sigma}\right)= \pm \sigma_{s}\left(x_{s} \alpha, x_{s} \beta, z_{s}\right)= \pm \sigma_{s} X_{s}, \\
& \underline{\chi}_{\tau}=\left(u \alpha_{\tau}, u \beta_{\tau}, 0\right)=(u / x)\left(x \alpha_{\tau}, x \beta_{\tau}, 0\right)=(u / x) X_{\tau} .
\end{aligned}
$$

Since $\underline{\chi}_{s}=d \underline{\chi}\left(X_{s}\right)$ and $\underline{\chi}_{\tau}=d \underline{\chi}\left(X_{\tau}\right)$ we see that $X_{s}$ and $X_{\tau}$ are eigenvectors of $d \underline{\chi}$ with eigenvalues $\pm \sigma_{s}$ and $\bar{u} \bar{x}$ respectively. The negative of the trace of $d \underline{\chi}$ is called the anisotropic mean curvature, which we will denote by $\Lambda$. In the case where $W$ is the 2-sphere, $\underline{\chi}$ is the Gauss map of $\Sigma$, and $\Lambda=2 H$, where $H$ is the usual mean curvature.

The anisotropic mean curvature, of course, has a more important variational definition. If an immersion $X$ is subjected to a normal variation $X_{\epsilon}=X+\epsilon \psi \nu+$ $\mathcal{O}\left(\epsilon^{2}\right)$ for $\psi \in C_{0}^{\infty}$, then the first variation of $\mathscr{F}$ is

$$
\partial_{\epsilon} \mathscr{F}\left(X_{\epsilon}\right)_{\epsilon=0}=-\int_{\Sigma} \psi \Lambda d \Sigma
$$

In particular, $\Lambda \equiv$ constant characterizes surfaces which are in equilibrium for the functional $\mathscr{F}$ subject to a volume constraint; see [Koiso and Palmer 2005].

From our computations, the anisotropic mean curvature is a constant $\Lambda$ if and only if

$$
\pm \sigma_{s}+u / x=-\Lambda=\text { constant }
$$

However, by (2-6), we have $u_{s}=u_{\sigma} \sigma_{s}= \pm x_{s} \sigma_{s}$ and so

$$
\frac{d u}{d x}=\frac{u_{s}}{x_{s}}= \pm \sigma_{s} .
$$

Therefore, (2-7) is equivalent to

$$
d u / d x+u / x=-\Lambda .
$$

Equation (2-9) is the same as $(x u)_{x}=-\Lambda x$. Integrating this equation gives

$$
u x=-\Lambda\left(x^{2} / 2\right)+c / 2
$$

where $c$ is a constant. We obtain

$$
x= \begin{cases}(-1 / \Lambda)\left(u \pm \sqrt{u^{2}+\Lambda c}\right) & \text { if } \Lambda \neq 0, \\ c /(2 u) & \text { if } \Lambda=0 .\end{cases}
$$


Assume $(\Lambda, c) \neq(0,0)$. Then, we can also use (2-6) to give a formula for $z$. We have, using (2-8),

$$
d z=(d z / d s) d s= \pm v_{\sigma} s_{\sigma} d \sigma=v_{\sigma} x_{u} d \sigma=x_{u} d v .
$$

The function $z$ can then be defined on a suitable subset of $W$ as

$$
z:=\int x_{u} d v
$$

with $x_{u}$ computed from (2-11). When inserted in (2-3), the functions $x$ defined by (2-11) and $z$ defined by (2-13) define an immersion on a suitable subset of $W$ which has constant anisotropic mean curvature $\Lambda$.

Let us consider the case where $(\alpha, \beta)=(\cos \tau, \sin \tau)$, that is, $W$ is a smooth, closed convex surface of revolution generated by the curve $\Omega_{W}$ in (2-1). The surfaces $X(s, \tau)$ of revolution with constant anisotropic mean curvature $\Lambda$ were studied in detail in [Koiso and Palmer 2005; 2006; 2007a; 2007b]. We refer to them as anisotropic Delaunay surfaces. In the special case where $W$ is the 2sphere, the surfaces $X(s, \tau)$ are just the usual Delaunay surfaces.

Denote by $\mu_{2}$ the principal curvature of the Wulff shape $W$ along the "equator" with respect to the inward pointing normal. Then $\mu_{2}=v_{\sigma} / u$, and therefore,

$$
\mu_{2}= \pm z^{\prime} / u \text {. }
$$

The curvature $\mu_{2}$ can be regarded as a function of the unit normal $v=\left(v_{1}, v_{2}, v_{3}\right)=$ $\left(z^{\prime} \cos \tau, z^{\prime} \sin \tau,-x^{\prime}\right)$. In the present case, $\mu_{2}$ can be regarded as a function of one variable: $\mu_{2}=\mu_{2}\left(v_{3}\right)=\mu_{2}\left(-x^{\prime}\right)$. Then (2-10) with (2-14) implies $\pm 2 \mu_{2}^{-1} z^{\prime} x+$ $\Lambda x^{2}=c$. The constant of integration $c$ will be called the flux parameter.

Let $S$ be an anisotropic Delaunay surface for the Wulff shape $W$. Let $\hat{S}$ be a reflection of $S$ with respect to a horizontal plane. Then $\hat{S}$ is also an anisotropic Delaunay surface for the same Wulff shape $W$. If the anisotropic mean curvature of $S$ is $\Lambda$ for the "outward" pointing normal, then the anisotropic mean curvature of $\hat{S}$ is $-\Lambda$ for the "inward" pointing normal. Therefore, from now on, we will identify an anisotropic Delaunay surface with its reflection with respect to a horizontal plane, and we may assume that $\Lambda \leq 0$. We remark that, for the CMC (constant mean curvature) case, this normalization for the sign of $\Lambda$ corresponds to a suitable choice of the orientation of the surface normal.

Now, we may consider only solutions of

$$
2 \mu_{2}^{-1} z^{\prime} x+\Lambda x^{2}=c
$$

with $\Lambda \leq 0$. Then the anisotropic Delaunay surfaces fall into six cases as follows:

(I-1) $\quad \Lambda=0$ and $c=0$ : horizontal plane.

(I-2) $\quad \Lambda=0$ and $c \neq 0$ : anisotropic catenoid. 
(II-1) $\Lambda<0$ and $c=0$ : Wulff shape (up to vertical translation and homothety).

(II-2) $\Lambda<0$ and $c=\left(\left(\left.\mu_{2}\right|_{\nu_{3}=0}\right)^{2}|\Lambda|\right)^{-1}$ : vertical cylinder.

(II-3) $\Lambda<0$ and $\left(\left(\left.\mu_{2}\right|_{v_{3}=0}\right)^{2}|\Lambda|\right)^{-1}>c>0$ : anisotropic unduloid.

(II-4) $\Lambda<0$ and $c<0$ : anisotropic nodoid.

The global behavior of these surfaces is strikingly similar to that of the classical Delaunay surfaces. For example, an anisotropic unduloid is a periodic embedded surface while an anisotropic nodoid is a periodic surface with self-intersections. The reader is referred to [Koiso and Palmer 2005, Section 5] for details. Here we note only the correspondence between these classes and the formulas for $x$ and $z$ given before. The case (I-2) corresponds to $(x, z)$ defined by (2-11) with $\Lambda=0$ and (2-13). The case (II-4) is obtained with $(x, z)$ defined by (2-11) with $\Lambda \neq 0$ for $-u_{0} \leq u \leq u_{0}$ with the + sign and (2-13), where $u_{0}:=\max u=u(0)=$ $-u\left(-2 L_{1}\right)=-u\left(2 L_{2}\right)$. The part of the surface defined in $u>0$ corresponds to where the Gaussian curvature is positive; where $u<0$, the Gaussian curvature is negative. Case (II-3) is obtained by using (2-11) (with $\Lambda \neq 0$ ) and (2-13) for $\sqrt{-\Lambda c} \leq u \leq u_{0}$. The part of the surface obtained using the + sign in (2-11) corresponds to where the Gaussian curvature is positive, whereas the part obtained using the - sign has negative curvature. The two parts can be smoothly joined together. Also, we remark that now

$$
x_{s}=u_{\sigma} \quad \text { and } \quad z_{s}=v_{\sigma} \quad \text { at } \sigma=\sigma(s) .
$$

Finally, it should be pointed out that, if we adopt $\mu_{2}=v_{\sigma} / u$ as the definition of $\mu_{2}$, then the calculation of the curve $(x, z)$ from $W$ only depended on the curve $(u, v)$. Therefore the curve $(\alpha, \beta)$ can be simultaneously varied in both the formulas for $X$ and $\chi$ without affecting the conclusion. These surfaces $X$ have similar classification and properties to those of the anisotropic Delaunay surfaces mentioned above. We will call these surfaces generalized anisotropic Delaunay surfaces. Examples of some generalized anisotropic Delaunay surfaces and the corresponding Wulff shapes are shown in Figures 1 through 4.

We conclude this section with a remark about the relationship between harmonic maps and anisotropic Delaunay surfaces.

Proposition 2.1. For any anisotropic Delaunay surface $X: \Sigma \rightarrow \mathbb{R}^{3}$, the anisotropic Gauss map $\underline{\chi}: \Sigma \rightarrow W$ is harmonic. In particular, when the surface is an anisotropic catenoid or the Wulff shape, $\underline{\chi}$ is \pm holomorphic.

Proof. The metric induced by $X(s, \tau)=(x(s) \cos \tau, x(s) \sin \tau, z(s))$ is $d S_{X}^{2}=$ $d s^{2}+x^{2} d \tau^{2}$. We can introduce isothermal coordinates $(\rho, \tau)$ for this metric by defining $d \rho=d s / x$ so that $d S_{X}^{2}=x^{2}\left(d \rho^{2}+d \tau^{2}\right)$. 

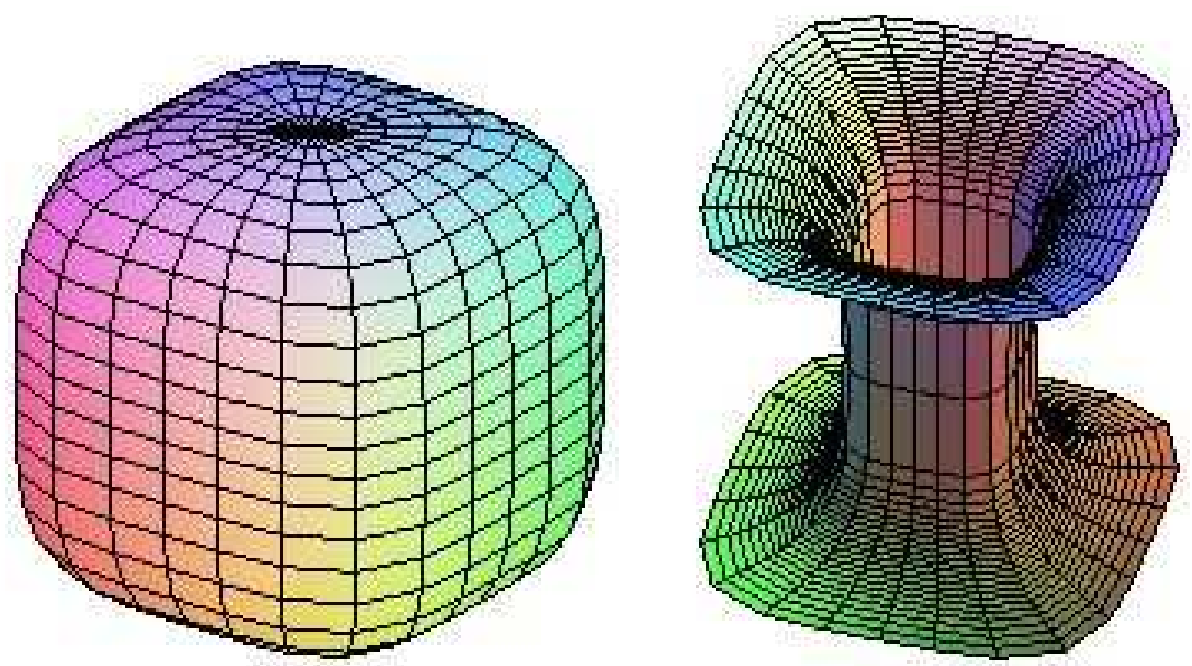

Figure 1. The Wulff shape at left is the superellipsoid $u^{4}+v^{4}+$ $w^{4}=1$. At right, its generalized anisotropic catenoid, for $c=1$.
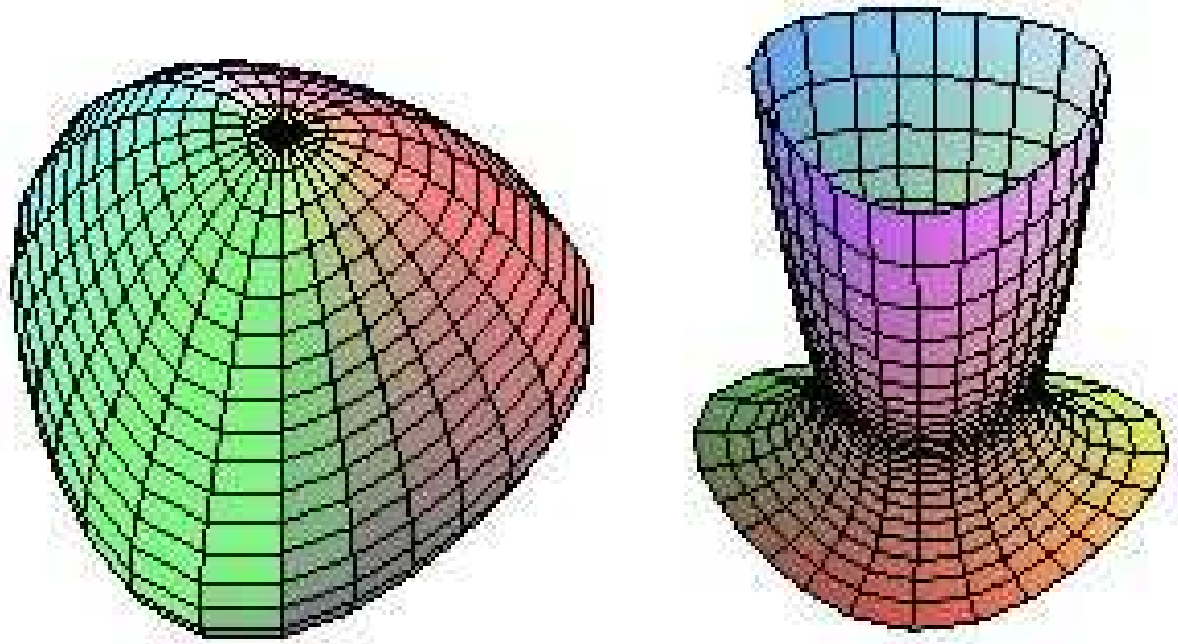

Figure 2. Left: this Wulff shape is generated from the superformula of [Gielis 2003], $r=\left\{\left|\cos \left(\frac{m \theta}{4}\right)\right|^{n_{2}}+\left|\sin \left(\frac{m \theta}{4}\right)\right|^{n_{3}}\right\}^{-n_{1}}$ with $\left(m, n_{1}, n_{2}, n_{3}\right)=(3,3.2,3,3)$. Right: its generalized anisotropic catenoid.

The induced metric on $W$ is $d S_{W}^{2}=d \sigma^{2}+u^{2} d \tau^{2}$. Then

$$
\begin{aligned}
\underline{\chi}^{*}\left(d S_{W}^{2}\right)=\sigma_{s}^{2} d s^{2}+u^{2} d \tau^{2} & =(-\Lambda-u / x)^{2} x^{2} d \rho^{2}+u^{2} d \tau^{2} \\
& =(-\Lambda x-u)^{2} d \rho^{2}+u^{2} d \tau^{2} .
\end{aligned}
$$



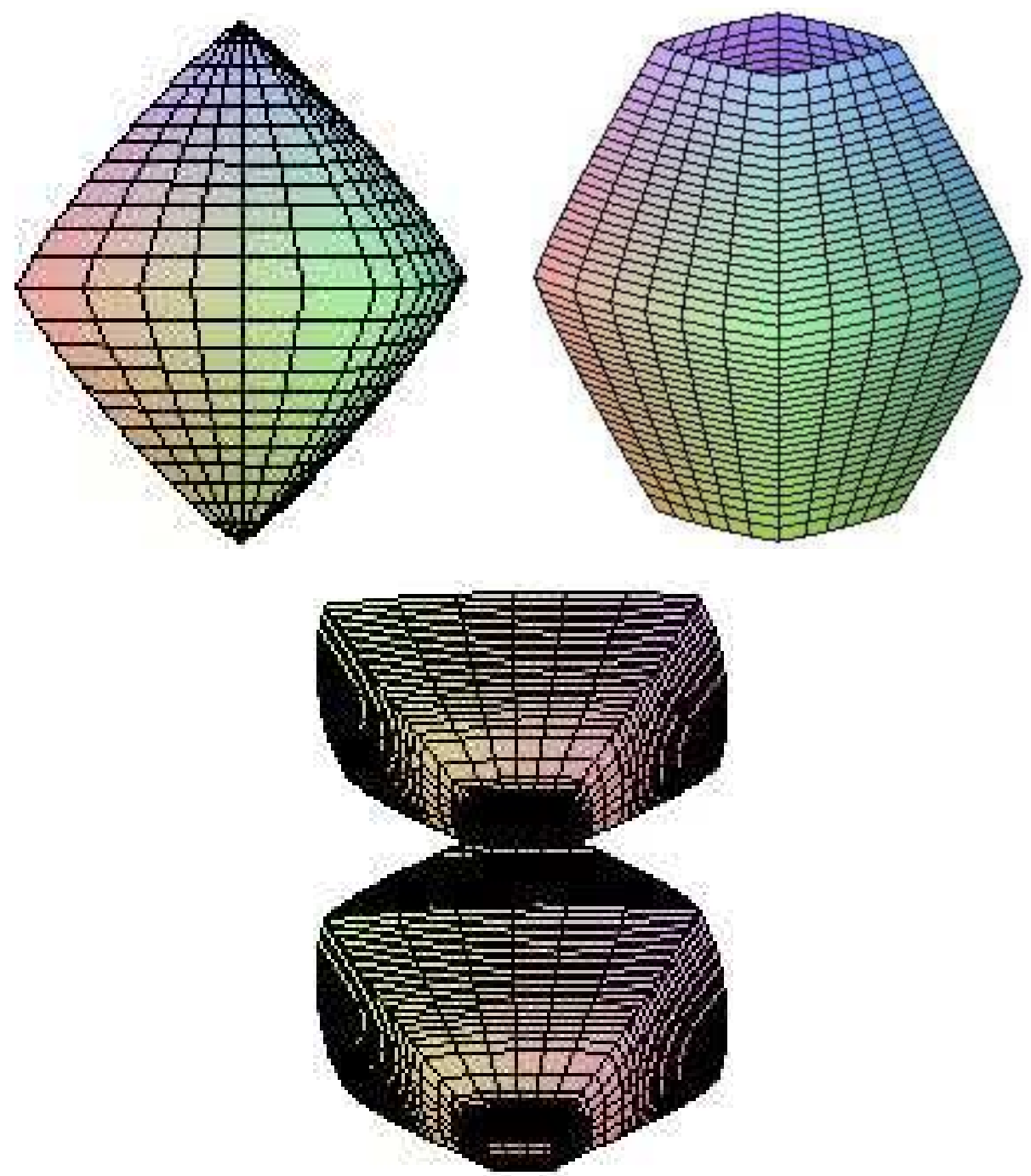

Figure 3. Left: the Wulff shape is the subellipsoid $|u|^{8 / 7}+|v|^{8 / 7}+$ $|w|^{8 / 7}=1$. Right: the convex part of its generalized anisotropic unduloid. $\Lambda=-0.5$ and $c=1$. Below, more of the same surface.

We recall that a sufficient condition for the harmonicity of a map from a surface with isothermal coordinates $(\rho, \tau)$ is that the pull back of the metric on the target space can be expressed as $E d \rho^{2}+2 F d \rho d \tau+G d \tau^{2}$ with $(E-G)-2 i F$ holomorphic as a function of $\rho+i \tau$.

In our case, we have

$$
(E-G)-2 i F=(-\Lambda x-u)^{2}-u^{2}=\Lambda\left(2 u x+\Lambda x^{2}\right)=\Lambda c=\text { constant }
$$




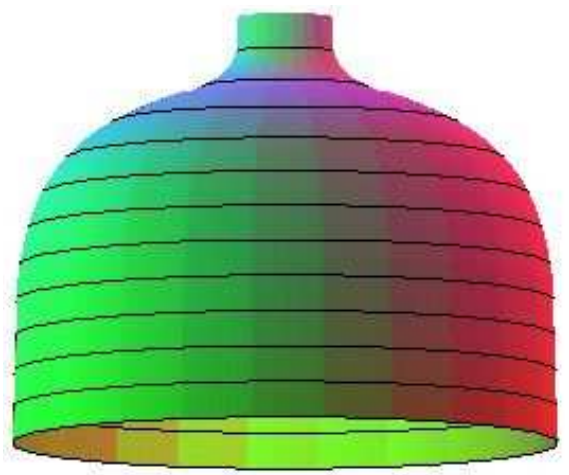

Figure 4. A half period of an anisotropic unduloid with Wulff shape $u^{4}+v^{4}=1$.

by (2-10), and so this function is holomorphic. In the case of the anisotropic catenoid $(\Lambda=0)$ or the Wulff shape $(c=0)$, this function vanishes, and we obtain the stronger result that $\underline{\chi}$ is \pm holomorphic.

It does not appear that the anisotropic Gauss map is harmonic if the anisotropic Delaunay surface is replaced by a general constant anisotropic mean curvature surface.

\section{Rolling construction for general curves}

We consider a smooth curve

$$
\Omega(s)=(x(s), z(s)) \quad \text { with } \quad x>0 \text { for } 0 \leq s \leq l,
$$

represented by the arc length $s$. The curve $\Omega$ will always be regarded as the generating curve of a surface of revolution $S: X(s, t)=\left(x(s) e^{i t}, z(s)\right)$ with respect to the $z$-axis, where we identify $\mathbb{C} \times \mathbb{R}$ with $\mathbb{R}^{3}$. Our point of departure will be [Hsiang and $Y u$ 1981], which investigates a rolling construction for general curves. We also refer the reader to [Guggenheimer 1977].

We will consider a configuration consisting of the following:

- The curve $\Omega$ itself, which in the current context will be called the roulette.

- A second curve $\Gamma$ which will be called the rolling curve.

- A point $P$ called the pole, whose location relative to $\Gamma$ is kept fixed when $\Gamma$ is moved. We express this by saying that $P$ is stationary with respect to $\Gamma$.

- A fixed line $L$ called the base, which is somewhere tangent to $\Gamma$.

According to [Hsiang and Yu 1981], away from umbilic points of the surface $X$, there exists a configuration as above, such that $\Omega$ is obtained as the trace of the 
pole as $\Gamma$ is rolled without slipping along the line $L$. A classical example is given by taking $\Omega$ to be the generating curve of a catenoid $S, \Gamma$ to be the parabola such that the distance between the vertex and the focus is equal to the distance between the vertex of $\Omega$ and the rotation axis of $S$, and $P$ to be the focus of $\Gamma$. Using formulas from [Hsiang and Yu 1981], it follows that the curve $\Gamma$ is given in polar coordinates $r=r(\theta)$ around the pole by

$$
\begin{aligned}
& r=\frac{x}{\left|z^{\prime}\right|} \\
& \theta=-\int\left(\frac{z^{\prime}}{x}-\kappa\right) d s=\int\left(k_{2}-k_{1}\right) d s,
\end{aligned}
$$

where

$$
\kappa:=x^{\prime} z^{\prime \prime}-x^{\prime \prime} z^{\prime}, \quad k_{1}=x^{\prime \prime} z^{\prime}-x^{\prime} z^{\prime \prime}, \quad k_{2}=-x^{-1} z^{\prime} .
$$

Here $\kappa$ is the curvature of the plane curve $(x(s), z(s))$, and $k_{1}$ are $k_{2}$ are the principal curvatures of $S$ with respect to the outward normal if $z^{\prime}>0$, and with respect to the inward normal if $z^{\prime}<0$.

Recall that a surface $S$ is called isothermic if, away from umbilic points, its lines of curvature are given as the level sets of a pair of locally defined conjugate harmonic functions. CMC surfaces and surfaces of revolution are examples of isothermic surfaces.

According to a classical theorem of Bour and Christofell, see [Eisenhart 1960], to each isothermic surface corresponds an isothermic dual $\tilde{S}$, defined up to homothety and translation, with the property that $S$ and $\tilde{S}$ are anticonformal and they share the same Gauss map. The construction of the isothermic dual involves integration and is therefore only well defined, in general, over simply connected domains. In the case of an anisotropic Delaunay surface, the duality is global, as Lemmas 7.1, 7.2, 7.4 and 7.5 below will show.

Another familiar case of isothermic duality involves a nonspherical surface $X$ with constant mean curvature $H \neq 0$. In this case, the parallel CMC surface $\tilde{X}:=$ $H X+v$ is the isothermic dual. Although $\tilde{X}$ will have branch points when $X$ has umbilics, it will however be globally defined.

In the case of a surface of revolution, the isothermic dual is given by the formulas in the following proposition.

Proposition 3.1. Set

$$
\tilde{x}(s):=\frac{a}{x(s)} \quad \text { and } \quad \tilde{z}(s):=-a \int_{0}^{s} \frac{z^{\prime}}{x^{2}} d s+b,
$$

where $a$ is a nonzero constant and $b$ is any constant. Then the surface $\tilde{S}: \tilde{X}(s, t)=$ $\left(\tilde{x}(s) e^{i t}, \tilde{z}(s)\right)$ is an isothermic dual of $S: X(s, t)=\left(x(s) e^{i t}, z(s)\right)$. 
Remark 3.1. When the generating curve $(x, z)$ is closed, the isothermic dual need not be globally defined because of a period problem. This occurs for example when $S$ is the Clifford torus generated by rotating the circle of radius one and center $(\sqrt{2}, 0)$ about a vertical axis.

Proof of Proposition 3.1.. We have

$$
\tilde{x}^{\prime}=-a x^{\prime} / x^{2} \text { and } \tilde{z}^{\prime}=-a z^{\prime} / x^{2} .
$$

Therefore,

$$
\begin{aligned}
\left(\tilde{x}^{\prime}, \tilde{z}^{\prime}\right) & =-\frac{a}{x^{2}}\left(x^{\prime}, z^{\prime}\right), \\
d \tilde{s} & :=\sqrt{(d \tilde{x})^{2}+(d \tilde{z})^{2}}=\sqrt{\left(\tilde{x}^{\prime}\right)^{2}+\left(\tilde{z}^{\prime}\right)^{2}} d s=\frac{|a|}{x^{2}} d s, \\
\left(\tilde{x}_{\tilde{s}}, \tilde{z}_{\tilde{s}}\right) & = \begin{cases}\left(-x^{\prime},-z^{\prime}\right) & \text { for } a>0, \\
\left(x^{\prime}, z^{\prime}\right) & \text { for } a<0 .\end{cases}
\end{aligned}
$$

If we compute the induced metric $d \tilde{S}^{2}$ for the surface $\tilde{S}$, we see that

$$
d \tilde{S}^{2}=\tilde{x}^{2} d \tau^{2}+d \tilde{s}^{2}=\frac{a^{2}}{x^{4}}\left(x^{2} d \tau^{2}+d s^{2}\right)=\frac{a^{2}}{x^{4}} d S^{2},
$$

where $d S^{2}$ is the metric on $S$. Thus the metrics on the two surfaces are conformally related; see [Eisenhart 1960, page 388].

On the other hand, the Gauss map $v$ of $X$ is

$$
v:=\frac{X_{t} \times X_{s}}{\left|X_{t} \times X_{s}\right|}=(\operatorname{sgn} x)\left(z^{\prime} \cos t, z^{\prime} \sin t,-x^{\prime}\right),
$$

where $(\operatorname{sgn} x)$ is the sign of $x$. Also, by using (3-3), (3-4), and (3-5), the Gauss map $\tilde{v}$ of $\tilde{X}$ is

$$
\begin{aligned}
\tilde{v} & :=\frac{\tilde{X}_{t} \times \tilde{X}_{s}}{\left|\tilde{X}_{t} \times \tilde{X}_{s}\right|}=(\operatorname{sgn} \tilde{x}) \cdot \frac{\left(\tilde{z}^{\prime} \cos t, \tilde{z}^{\prime} \sin t,-\tilde{x}^{\prime}\right)}{\sqrt{\left(\tilde{x}^{\prime}\right)^{2}+\left(\tilde{z}^{\prime}\right)^{2}}} \\
& =-(\operatorname{sgn} x)\left(z^{\prime} \cos t, z^{\prime} \sin t,-x^{\prime}\right)=-v .
\end{aligned}
$$

Therefore, if we choose the orientation of the surface $\tilde{S}$ so that its Gauss map coincides with $v$, then the map $X(s, t) \mapsto \tilde{X}(s, t)$ is anticonformal.

We will see in Section 7 that, when $S$ is a Wulff shape, $\tilde{\Omega}$ generates an anisotropic catenoid, and when $S$ is an anisotropic catenoid, $\tilde{\Omega}$ generates a homothety of the Wulff shape. Also we will see that the isothermic dual of any anisotropic unduloid (respectively nodoid) is a part of the same surface, that is, the anisotropic unduloids (respectively nodoids) are self-dual. In fact, this self-duality will characterize these classes of curves without reference to the functional; see Theorem 8.1. 
Set

$$
\tilde{k}_{1}:=\tilde{x}_{\tilde{s}} \tilde{z}_{\tilde{s} \tilde{s}}-\tilde{x}_{\tilde{s} \tilde{s}} \tilde{z}_{\tilde{s}}, \quad \tilde{k}_{2}:=\frac{\tilde{z}_{\tilde{s}}}{\tilde{x}}, \quad \tilde{H}:=\left(\tilde{k}_{1}+\tilde{k}_{2}\right) / 2 .
$$

Then $\tilde{k}_{1}$ and $\tilde{k}_{2}$ are the principal curvatures and $\tilde{H}$ is the mean curvature of the surface $\left(\tilde{x}(\tilde{s}) e^{i t}, \tilde{z}(\tilde{s})\right)$ with respect to the outward pointing normal if $z^{\prime}>0$ and with respect to the inward pointing normal if $z^{\prime}<0$. We now compute

$$
\left(\frac{d^{2} \tilde{x}}{d \tilde{s}^{2}}, \frac{d^{2} \tilde{z}}{d \tilde{s}^{2}}\right)=-\frac{1}{a} x^{2}\left(x^{\prime \prime}, z^{\prime \prime}\right) .
$$

Hence, we obtain

$$
\tilde{k}_{1}=-\frac{x^{2}}{|a|} k_{1}, \quad \tilde{k}_{2}=\frac{x^{2}}{|a|} k_{2}, \quad \tilde{H}=x^{2}\left(k_{2}-k_{1}\right) /|2 a| .
$$

By (3-1), (3-2), (3-5), and (3-7), we obtain

$$
\begin{aligned}
& r=\left|\frac{a}{\tilde{x} \tilde{z}_{\tilde{s}}}\right|>0, \\
& \theta=\int\left(\tilde{k}_{1}+\tilde{k}_{2}\right) d \tilde{s}=2 \int \tilde{H} d \tilde{s}=\int\left(k_{2}-k_{1}\right) d s .
\end{aligned}
$$

Lemma 3.1. The rolling curve $\Gamma$ of a smooth curve

$$
\Omega(s)=(x(s), z(s)) \quad \text { with } \quad x(s)>0 \quad \text { and } \quad z^{\prime}(s) \neq 0
$$

is a regular curve away from umbilic points of the rotation surface $X(s, t)=$ $\left(x(s) e^{i t}, z(s)\right)$. In other words, the rolling curve $\Gamma$ is regular if $\tilde{H} \neq 0$. Moreover, at any nonumbilic point of $X$, the curvature $\kappa_{\Gamma}$ of $\Gamma$ is

$$
\kappa_{\Gamma}=-\frac{\left(z^{\prime}\right)^{3}}{x^{2}\left|k_{2}-k_{1}\right|}=-\frac{\left(z^{\prime}\right)^{3}}{2|a \tilde{H}|} .
$$

Proof. We represent the rolling curve $\Gamma: r=r(\theta)$ as

$$
\xi(s)=r(s) \cos \theta(s)+\xi_{0} \quad \text { and } \quad \eta(s)=r(s) \sin \theta(s)+\eta_{0} .
$$

Here, $s$ is arc length of the curve $\Omega=(x(s), z(s))$, but in general, it is not arc length of $\Gamma$. By elementary calculations, we obtain

$$
\begin{aligned}
\left(\xi^{\prime}\right)^{2}+\left(\eta^{\prime}\right)^{2}=\left(r^{\prime}\right)^{2}+r^{2}\left(\theta^{\prime}\right)^{2} & =\left(z^{\prime}-\kappa x\right)^{2}\left(z^{\prime}\right)^{-4} \\
& =x^{2}\left(k_{1}-k_{2}\right)^{2}\left(z^{\prime}\right)^{-4}=4 a^{2} \tilde{H}^{2} x^{-2}\left(z^{\prime}\right)^{-4} .
\end{aligned}
$$

This implies the first half of the lemma. 
We compute

$$
\begin{aligned}
\kappa_{\Gamma}:=\frac{\xi^{\prime} \eta^{\prime \prime}-\xi^{\prime \prime} \eta^{\prime}}{\left\{\left(\xi^{\prime}\right)^{2}+\left(\eta^{\prime}\right)^{2}\right\}^{3 / 2}} & =\frac{2\left(r^{\prime}\right)^{2} \theta^{\prime}+r r^{\prime} \theta^{\prime \prime}-r r^{\prime \prime} \theta^{\prime}+r^{2}\left(\theta^{\prime}\right)^{3}}{\left\{\left(r^{\prime}\right)^{2}+r^{2}\left(\theta^{\prime}\right)^{2}\right\}^{3 / 2}} \\
& =\frac{-\left(\kappa x-z^{\prime}\right)^{2}}{x\left(z^{\prime}\right)^{3}}\left(\frac{\left(\kappa x-z^{\prime}\right)^{2}}{\left(z^{\prime}\right)^{4}}\right)^{-3 / 2} \\
& =-\frac{\left(z^{\prime}\right)^{3}}{x\left|\kappa x-z^{\prime}\right|}=-\frac{\left(z^{\prime}\right)^{3}}{x^{2}\left|k_{2}-k_{1}\right|}=-\frac{\left(z^{\prime}\right)^{3}}{2|a \tilde{H}|} .
\end{aligned}
$$

Theorem 3.1. Let

$$
\Omega(s)=(x(s), z(s)) \quad \text { with } \quad x(s)>0 \quad \text { and } \quad z^{\prime}(s) \neq 0
$$

be a smooth curve with arc length $s$. Denote by $R(s)=\left(R_{x}(s), R_{z}(s)\right)$ the center of curvature of $\Omega$ at s. Set $X(s, t)=\left(x(s) e^{i t}, z(s)\right)$, which is the surface of revolution generated by $\Omega$ with z-axis as rotation axis. Denote by $\hat{k}_{1}$ and $\hat{k}_{2}$ the principal curvatures of $X$ with respect to the inward pointing normal. Here, $\hat{k}_{1}$ is the curvature of $\Omega$. Let $\Gamma$ be the rolling curve of $\Omega$ with $z$-axis as base. The following hold:

(I) If $\hat{k}_{1}\left(s_{0}\right) \neq \hat{k}_{2}\left(s_{0}\right)$, then $\Gamma$ is a regular smooth curve near $s=s_{0}$. The condition $\hat{k}_{1}\left(s_{0}\right) \neq \hat{k}_{2}\left(s_{0}\right)$ is equivalent to the condition that either $\hat{k}_{1}\left(s_{0}\right)=0$ or $R_{x}\left(s_{0}\right) \neq$ 0 .

(II) Assume $\hat{k}_{1}\left(s_{0}\right) \neq \hat{k}_{2}\left(s_{0}\right)$. Then $\Gamma \subset\{x>0\}$ (respectively $\Gamma \subset\{x<0\}$ ) for $0<\left|s-s_{0}\right|<\delta$ for some $\delta>0$ if and only if $\hat{k}_{2}\left(s_{0}\right)>\hat{k}_{1}\left(s_{0}\right)$ (respectively $\left.\hat{k}_{2}\left(s_{0}\right)<\hat{k}_{1}\left(s_{0}\right)\right)$.

(III) (i) Assume $\hat{k}_{1}\left(s_{0}\right)>0$, that is, the Gauss curvature of $X$ is positive near $s=s_{0}$. Then $R_{x}\left(s_{0}\right)<0$ (respectively $\left.R_{x}\left(s_{0}\right)>0\right)$ if and only if $\Gamma \subset\{x>0\}$ (respectively $\Gamma \subset\{x<0\}$ ) for $0<\left|s-s_{0}\right|<\delta$ for some $\delta>0$.

(ii) Assume $\hat{k}_{1}\left(s_{0}\right)<0$, that is, the Gauss curvature of $X$ is negative near $s=s_{0}$. Then, $R_{x}\left(s_{0}\right)>0$, and $\Gamma \subset\{x>0\}$ for $0<\left|s-s_{0}\right|<\delta$ for some $\delta>0$.

Proof. Assume that the rotation surface $\left(x(s) e^{i t}, z(s)\right)$ has no umbilic points. We represent the rolling curve $\Gamma: r=r(\theta)$ of $\Omega$ as

$$
\xi(s)=r(s) \cos \theta(s)+\xi_{0} \quad \text { and } \quad \eta(s)=r(s) \sin \theta(s)+\eta_{0} .
$$

Then $r=x /\left|z^{\prime}\right|$ and $\theta^{\prime}=k_{2}-k_{1}$, where $k_{1}=x^{\prime \prime} z^{\prime}-x^{\prime} z^{\prime \prime}$ and $k_{2}=-z^{\prime} / x$, and, by Lemma 3.1, the curvature $\kappa_{\Gamma}$ of $\Gamma$ is

$$
\begin{aligned}
\kappa_{\Gamma} & :=\frac{\xi^{\prime} \eta^{\prime \prime}-\xi^{\prime \prime} \eta^{\prime}}{\left\{\left(\xi^{\prime}\right)^{2}+\left(\eta^{\prime}\right)^{2}\right\}^{3 / 2}} \\
& =-\frac{\left(z^{\prime}\right)^{3}}{x^{2}\left|k_{2}-k_{1}\right|} .
\end{aligned}
$$


Let $Q:=\left(\xi\left(s_{0}\right), \eta\left(s_{0}\right)\right)$ be the point of contact between $\Gamma$ and the $z$-axis. Then, if $\eta^{\prime}\left(s_{0}\right)>0$, then, by (3-9), we have at $s=s_{0}$

$$
\begin{aligned}
& \kappa_{\Gamma}>0 \Longleftrightarrow \xi^{\prime \prime}<0 \Longleftrightarrow \Gamma \subset\{x<0\} \text { near } Q, \\
& \kappa_{\Gamma}<0 \Longleftrightarrow \xi^{\prime \prime}>0 \Longleftrightarrow \Gamma \subset\{x>0\} \text { near } Q .
\end{aligned}
$$

If $\eta^{\prime}\left(s_{0}\right)<0$, then, by (3-9), we have at $s=s_{0}$

$$
\begin{aligned}
& \kappa_{\Gamma}>0 \Longleftrightarrow \xi^{\prime \prime}>0 \Longleftrightarrow \Gamma \subset\{x>0\} \text { near } Q, \\
& \kappa_{\Gamma}<0 \Longleftrightarrow \xi^{\prime \prime}<0 \Longleftrightarrow \Gamma \subset\{x<0\} \text { near } Q .
\end{aligned}
$$

Also, by (3-10), we have at $s=s_{0}$

$$
\kappa_{\Gamma}>0 \Longleftrightarrow z^{\prime}<0 \quad \text { and } \quad \kappa_{\Gamma}<0 \Longleftrightarrow z^{\prime}>0 .
$$

On the other hand, if $z^{\prime}>0$, then $k_{1}$ and $k_{2}$ are principal curvatures of the surface $X(s, t)=\left(x(s) e^{i t}, z(s)\right)$ with respect to the outward pointing normal, whereas if $z^{\prime}<0$ they are the principal curvatures with respect to the inward pointing normal. Therefore,

$$
z^{\prime} \theta^{\prime}=z^{\prime}\left(k_{2}-k_{1}\right)=-\left|z^{\prime}\right|\left(\hat{k}_{2}-\hat{k}_{1}\right)=-x r^{-1}\left(\hat{k}_{2}-\hat{k}_{1}\right) .
$$

Note that $\eta^{\prime} \theta^{\prime}<0$ at $s=s_{0}$. Therefore, by (3-11) and (3-13), if $\theta^{\prime}\left(s_{0}\right)<0$, then

$$
\begin{aligned}
& z^{\prime}<0 \Longleftrightarrow \Gamma \subset\{x<0\} \text { near } Q, \\
& z^{\prime}>0 \Longleftrightarrow \Gamma \subset\{x>0\} \text { near } Q .
\end{aligned}
$$

By (3-12) and (3-13), if $\theta^{\prime}\left(s_{0}\right)>0$, then

$$
\begin{aligned}
& z^{\prime}<0 \Longleftrightarrow \Gamma \subset\{x>0\} \text { near } Q, \\
& z^{\prime}>0 \Longleftrightarrow \Gamma \subset\{x<0\} \text { near } Q .
\end{aligned}
$$

Therefore, if $\theta^{\prime} z^{\prime}\left(s_{0}\right)>0$ (respectively $\theta^{\prime} z^{\prime}\left(s_{0}\right)<0$ ), then $\Gamma \subset\{x<0\}$ (respectively $\Gamma \subset\{x>0\}$ ) for $0<\left|s-s_{0}\right|<\delta$ for some $\delta>0$. Hence, by (3-14), $\Gamma \subset\{x<0\}$ (respectively $\Gamma \subset\{x>0\}$ ) for $0<\left|s-s_{0}\right|<\delta$ for some $\delta>0$ if and only if $\hat{k}_{2}\left(s_{0}\right)<\hat{k}_{1}\left(s_{0}\right)$ (respectively $\hat{k}_{2}\left(s_{0}\right)>\hat{k}_{1}\left(s_{0}\right)$ ). We have proved (II).

By elementary calculations, we prove (I) by

$$
R=\left(R_{x}, R_{z}\right)=\left(x k_{1}^{-1}\left(k_{1}-k_{2}\right), z-k_{1}^{-1} x^{\prime}\right) .
$$

Lastly we prove (III). It is sufficient to prove it for the case where $z^{\prime}\left(s_{0}\right)>0$. In this case, $k_{1}=-\hat{k}_{1}$ and $k_{2}=-\hat{k}_{2}$. Now (III) follows from (II) and (3-15).

The rolling construction still applies when $S$ has umbilic points. Figure 5 depicts the rolling curve for the surface $S$ generated by the curve $u^{4}+v^{4}=1$. The cusps of the rolling curve correspond to the umbilics of $S$. We investigate this next. 

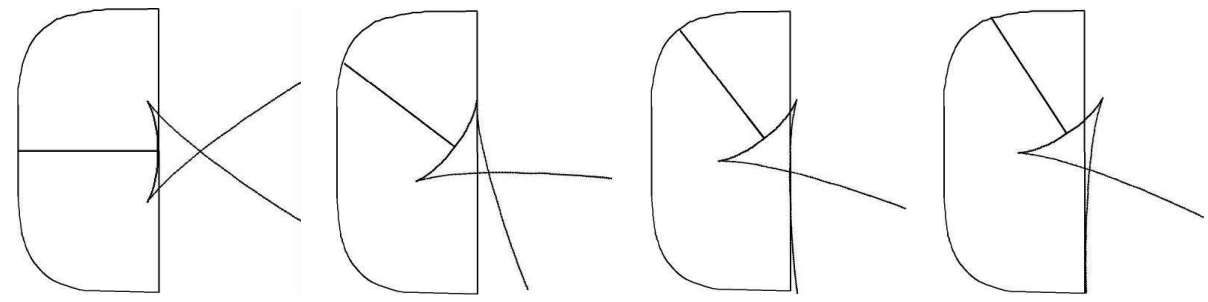

Figure 5. This series of pictures demonstrates the rolling construction for the Wulff shape whose generating curve is given by $u^{4}+v^{4}=1$.

Proposition 3.2. Let $\Omega$ and $\Gamma$ be the same as in Theorem 3.1. Let $s_{0}$ be an isolated zero of $\eta(s):=k_{1}-k_{2}$. Assume that $z^{\prime}\left(s_{0}\right) \neq 0$, that is, $r$ is bounded as $s \rightarrow s_{0}$ by (3-1). Then, the one sided limits

$$
\lambda_{ \pm}:=\lim _{s \rightarrow s_{0} \pm 0} \frac{\Gamma^{\prime}(s)}{\left\|\Gamma^{\prime}(s)\right\|}
$$

both exist. In addition, if $\eta(s)$ does not change sign at $s_{0}$, then $\lambda_{-}=\lambda_{+}$. If $\eta(s)$ changes sign at $s_{0}$, then $\lambda_{-}=-\lambda_{+}$and consequently, at $\Gamma\left(s_{0}\right)$, the limits of the unit tangent vectors to the two arcs exist and point in opposite directions.

Proof. A straightforward calculation using (3-1) and (3-2) shows that, for $s \approx s_{0}$, with $s \neq s_{0}$ and $\sigma:=\operatorname{sgn} z^{\prime}$, we have $r^{\prime}=\sigma\left(k_{1}-k_{2}\right)\left(x x^{\prime}\right) /\left(z^{\prime}\right)^{2}$. Therefore

$$
r^{\prime}+i \theta^{\prime} r=\sigma\left(k_{1}-k_{2}\right)\left(\frac{x x^{\prime}}{\left(z^{\prime}\right)^{2}}-i \frac{x}{z^{\prime}}\right),
$$

and so

$$
\frac{\Gamma^{\prime}}{\left\|\Gamma^{\prime}\right\|}=\rho \sigma\left(\frac{x x^{\prime}}{\left(z^{\prime}\right)^{2}}-i \frac{x}{z^{\prime}}\right) e^{i \theta}\left\|\frac{x x^{\prime}}{\left(z^{\prime}\right)^{2}}-i \frac{x}{z^{\prime}}\right\|^{-1},
$$

where $\rho=1$ if $k_{1}-k_{2}$ is positive, and $\rho=-1$ if $k_{1}-k_{2}$ is negative. Here $\theta(s)$

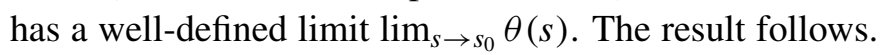

Remark 3.2. It follows from the previous proposition that the part of the rolling curve near $s=s_{0}$ consists of two $C^{1}$ arcs that lie on opposite (respectively the same) sides of the tangent line to $\Gamma$ if $k_{1}-k_{2}$ changes sign (respectively does not change sign) at $s_{0}$.

\section{Mean curvature profile}

Let $S: X(s, t)=\left(x(s) e^{i t}, z(s)\right)$ be a surface of revolution generated by a smooth curve $\Omega(s)=(x(s), z(s))$ parametrized by arc length. We assume that either $x(s)>$ 0 for all $s$ or $x(s)<0$ for all $s$. We set

$$
\hat{k}_{1}:=x^{\prime} z^{\prime \prime}-x^{\prime \prime} z^{\prime}, \quad \hat{k}_{2}:=\frac{z^{\prime}}{x}, \quad H_{S}:=\left(\hat{k}_{1}+\hat{k}_{2}\right) / 2 .
$$


$H_{S}(s)$ is the mean curvature of $S$ measured along the meridian $\Omega(s)$ with respect to the inward pointing normal if $x z^{\prime}>0$ and with respect to the outward pointing normal if $x z^{\prime}<0$.

The mean curvature profile of $S$ is the plane curve $C_{S}(s)=(f(s), g(s))$ defined by the properties

(A) $s$ is also the arc length parameter of $C_{S}$ and

(B) the curvature $\kappa_{C_{S}}(s)$ of $C_{S}$ is $\kappa_{C_{S}}(s)=2 H_{S}(s)$.

Note that the mean curvature profile is only determined up to rigid motion. The mean curvature profile was used extensively by Kenmotsu [1980; 2003] to study surfaces of revolution with prescribed mean curvature.

It is elementary that, up to rotation, the mean curvature profile $(f, g)$ is given by

$$
f(s):=\int_{0}^{s} \cos \theta\left(s_{1}\right) d s_{1}-c_{1} \quad \text { and } \quad g(s):=\int_{0}^{s} \sin \theta\left(s_{1}\right) d s_{1}-c_{2},
$$

where

$$
\theta(s):=2 \int_{0}^{s} H_{S}(s) d s,
$$

and $c_{1}$ and $c_{2}$ are constants. Kenmotsu [1980] showed that one can determine constants $c_{1}$ and $c_{2}$ so that

$$
\begin{aligned}
& x=(\operatorname{sgn} x) \sqrt{f^{2}+g^{2}}, \\
& z^{\prime}=(\operatorname{sgn} x) \frac{f g^{\prime}-f^{\prime} g}{\sqrt{f^{2}+g^{2}}} .
\end{aligned}
$$

The equality (4-3) can be expressed as follows:

(C) The distance between a point of $\Omega$ and the $z$-axis is equal to the distance between the corresponding point of $C_{S}$ and the origin.

(A), (B), and (C) determine the curve $C_{S}(s)=(f(s), g(s))$ up to rotation around the origin.

We can characterize the mean curvature profile in another way:

Proposition 4.1. Let $C_{S}(s)=(f(s), g(s))$ be a plane curve defined by the above properties $(A)$ and $(C)$. Then, $(B)$ is automatically satisfied (up to sign). The curve $C_{S}(s)=(f(s), g(s))$ is determined up to rotation around the origin. 
Proof. Express the curve $C_{S}(s)=(f(s), g(s))$ by the polar coordinate $(\rho, t)$, that is, $f=\rho \cos t$ and $g=\rho \sin t$. We regard $\rho, t$ as functions of $s$. Then,

$$
\begin{aligned}
\kappa_{C_{S}}(s) & :=\frac{f^{\prime} g^{\prime \prime}-f^{\prime \prime} g^{\prime}}{\left\{\left(f^{\prime}\right)^{2}+\left(g^{\prime}\right)^{2}\right\}^{3 / 2}} \\
& =\frac{2\left(\rho^{\prime}\right)^{2} t^{\prime}+\rho \rho^{\prime} t^{\prime \prime}-\rho \rho^{\prime \prime} t^{\prime}+\rho^{2}\left(t^{\prime}\right)^{3}}{\left\{\left(\rho^{\prime}\right)^{2}+\rho^{2}\left(t^{\prime}\right)^{2}\right\}^{3 / 2}} .
\end{aligned}
$$

Conditions (A) and (C) respectively imply

$$
d x^{2}+d z^{2}=d \rho^{2}+\rho^{2} d t^{2} \quad \text { and } \quad|x|=\rho .
$$

Together, these imply $d z^{2}=x^{2} d t^{2}$, and therefore

$$
t^{\prime}= \pm \frac{z^{\prime}}{x} \quad \text { and } \quad t^{\prime \prime}= \pm \frac{z^{\prime \prime} x-z^{\prime} x^{\prime}}{x^{2}}
$$

Inserting (4-7) and the second of (4-6) into (4-5), we obtain

$$
\kappa_{C_{S}}(s)= \pm\left\{\left(x^{\prime} z^{\prime \prime}-x^{\prime \prime} z^{\prime}\right)+\frac{z^{\prime}}{x}\right\}= \pm 2 H_{S}(s) .
$$

When $(f, g)$ satisfies (A), (B), and (C), we call the curve $(f, g)$ the mean curvature profile associated with $S$.

Lemma 4.1. If $z^{\prime}(0)= \pm 1$, then $c_{1}=0$ and $c_{2}=\left(\operatorname{sgn} z^{\prime}(0)\right) x(0)$.

Proof. At $s=0$, we have

$$
\begin{gathered}
f(0)=-c_{1}, \quad f^{\prime}(0)=1, \quad g(0)=-c_{2}, \quad g^{\prime}(0)=0, \\
x(0)=(\operatorname{sgn} x(0)) \sqrt{c_{1}^{2}+c_{2}^{2}}, \quad z^{\prime}(0)=(\operatorname{sgn} x(0)) c_{2} / \sqrt{c_{1}^{2}+c_{2}^{2}} .
\end{gathered}
$$

These equalities imply the result.

\section{Rolling curves as dual curves}

Let $\gamma=\gamma(s)=(f(s), g(s))$ be a smooth curve with arc length $s$ in the plane with unit normal $N$. Let $p:=\langle\gamma, N\rangle$ be the support function. Away from points where $N^{\prime}(s)=0$, that is, where the curvature $\kappa_{\gamma}$ vanishes at $s$, we can consider $p$ to be (locally) a function on a subset of the unit circle $S^{1}$. We assume that $p$ has at most isolated zeros. The curve $\gamma$ can be expressed using its tangential representation,

$$
\gamma=p N+D p
$$

where $D p$ is the gradient of $p$ on $S^{1}$. If $N=(\cos \theta, \sin \theta)$, then

$$
\gamma=(p \cos \theta-\dot{p} \sin \theta, p \sin \theta+\dot{p} \cos \theta), \quad \text { where } \dot{p}=d p / d \theta \text {. }
$$


We define the dual curve by $\gamma^{*}:=p^{-1} N$. Since

$$
\left(\gamma^{*}\right)^{\prime}=-p^{\prime} p^{-2} N+p^{-1} N^{\prime} \quad \text { and } \quad\left\langle N, N^{\prime}\right\rangle=0,
$$

$\gamma^{*}$ is a regular smooth curve around $s$ where $p(s) \neq 0$ and $\left(p^{\prime}(s), \kappa_{\gamma}(s)\right) \neq(0,0)$.

When $\gamma$ is the profile curve for the unit sphere of a rotationally invariant norm, then $\gamma^{*}$ is the profile curve of the unit sphere of the dual norm. Note that the curve $\gamma^{*}$ depends on the choice of origin in the plane.

$\gamma^{*}$ is characterized by the conditions $\left\langle d \gamma, \gamma^{*}\right\rangle=0$ and $\left\langle\gamma, \gamma^{*}\right\rangle \equiv 1$. Note that $\gamma^{* *}=\gamma$. This can be seen by differentiating the second condition to get $0=d 1=$ $d\left\langle\gamma, \gamma^{*}\right\rangle=\left\langle d \gamma, \gamma^{*}\right\rangle+\left\langle\gamma, d \gamma^{*}\right\rangle$. Thus the conditions still hold when $\gamma$ and $\gamma^{*}$ are interchanged.

Example 5.1. Let $\gamma$ be the circle of radius $\rho$ and center $(\epsilon, 0)$, where $\epsilon \geq 0$. Then,

$$
\begin{gathered}
\gamma=(\epsilon+\rho \cos \theta, \rho \sin \theta), \quad N=(\cos \theta, \sin \theta), \quad p=\rho+\epsilon \cos \theta, \\
\gamma^{*}=(1 / p) N=\frac{(1 / \rho)}{1+(\epsilon / \rho) \cos \theta}(\cos \theta, \sin \theta) .
\end{gathered}
$$

This means that $\gamma^{*}$ is a curve with polar equation $r=(1 / \rho) /(1+(\epsilon / \rho) \cos \theta)$. These equations describe all the various conic sections if one of the foci is at the origin, the directrix is the line $x=1 / \epsilon$, and $\epsilon / \rho$ is the eccentricity.

In the present context it is natural to treat all conic sections in a unified way. To do this, we consider the projective plane

$$
P^{2}(\mathbb{R}):=\left\{X=\left(x_{1}, x_{2}, x_{3}\right) \in \mathbb{R}^{3} ; X \neq(0,0,0)\right\} / \sim,
$$

where $\sim$ is the equivalence relation defined as follows: $\left(x_{1}, x_{2}, x_{3}\right) \sim\left(y_{1}, y_{2}, y_{3}\right)$ if and only if $\left(y_{1}, y_{2}, y_{3}\right)=c\left(x_{1}, x_{2}, x_{3}\right)$ for some $c \in \mathbb{R}$. Let $(\xi, \eta)$ be the orthogonal coordinates in the plane where the curve $\gamma$ lies. We identify each point $(\xi, \eta)$ in this plane with $(\xi, \eta, 1)$ in $P^{2}(\mathbb{R})$ and regard $\gamma$ as a curve in $P^{2}(\mathbb{R})$.

Lemma 5.1. Assume that $\kappa_{\gamma}(s) \neq 0$ for all $s$ in the domain of $\gamma$. Assume also that $p=\langle\gamma, N\rangle$ has at most isolated zeros. Then, the dual curve $\gamma^{*}$ can be regarded as a connected regular smooth curve in the projective plane $P^{2}(\mathbb{R})$.

Proof. Set $\gamma^{*}(s)=(\varphi(s), \psi(s))$. Then

$$
p=f g^{\prime}-f^{\prime} g, \quad \varphi=\frac{g^{\prime}}{f g^{\prime}-f^{\prime} g}, \quad \psi=-\frac{f^{\prime}}{f g^{\prime}-f^{\prime} g} .
$$

Therefore, at each point $s$ (including a point $s$ where $p(s)=0$ ),

$$
\gamma^{*}(s)=\left(g^{\prime},-f^{\prime}, f g^{\prime}-f^{\prime} g\right)
$$

in $P^{2}(\mathbb{R})$. Hence

$$
\left(\gamma^{*}\right)^{\prime}(s)=\left(g^{\prime \prime},-f^{\prime \prime}, f g^{\prime \prime}-f^{\prime \prime} g\right)=\kappa_{\gamma}\left(f^{\prime}, g^{\prime}, f f^{\prime}+g g^{\prime}\right) \neq\left(\gamma^{*}\right)(s)
$$


in $P^{2}(\mathbb{R})$. Therefore, $\gamma^{*}(s)$ is well defined for all $s$ and is a connected regular smooth curve in the projective plane $P^{2}(\mathbb{R})$.

For any smooth curve

$$
\Omega(s)=(x(s), z(s)) \quad \text { with } x>0 \text { and } z^{\prime}>0 \text { for } l_{1} \leq s \leq l_{2}
$$

$\left(l_{1} \leq 0<l_{2}\right)$ represented by the arc length $s$, and for a real constant $a \neq 0$, we recall from Section 3 that the isothermic dual is

$$
\tilde{x}(s):=\frac{a}{x(s)} \text { and } \quad \tilde{z}(s):=-a \int_{0}^{s} \frac{z^{\prime}}{x^{2}} d s .
$$

Now represent the coordinate functions of the rolling curve $\Gamma: r=r(\theta)$ of $\Omega$ as

$$
\xi(s)=r(s) \cos \theta(s)+\xi_{0} \quad \text { and } \quad \eta(s)=r(s) \sin \theta(s)+\eta_{0},
$$

where the point $\left(\xi_{0}, \eta_{0}\right)$ is the pole of $\Gamma$. Then, by elementary calculation, we obtain that the dual curve $\Gamma^{*}$ of $\Gamma$ is

$$
\Gamma^{*}=(1 / r)(\cos \theta, \sin \theta)+(1 / r)_{\theta}(-\sin \theta, \cos \theta) .
$$

We compute using (3-1) and (3-2) and that $s$ is the arc length of $(x, z)$, obtaining $r_{\theta}=-r x^{\prime} / z^{\prime}$. Therefore, from (3-6), we get $(1 / r)_{\theta}=\tilde{x}_{\tilde{z}} / r$. Thus

$$
\Gamma^{*}=(1 / r)\left[(\cos \theta, \sin \theta)+\tilde{x}_{\tilde{z}}(-\sin \theta, \cos \theta)\right] .
$$

Write $\tilde{x}_{\tilde{s}}=\cos \vartheta$ and $\tilde{z}_{\tilde{s}}=\sin \vartheta$; then

$$
\Gamma^{*}=\left(1 /\left(r \tilde{z}_{\tilde{s}}\right)\right)(\sin (\vartheta-\theta), \cos (\vartheta-\theta)) .
$$

This can be expressed, because of (3-6), (3-8), (5-1), and (5-2), as

$$
\Gamma^{*}=(-1 /|a|) \tilde{x} \cdot(\sin (\vartheta-\theta), \cos (\vartheta-\theta)) .
$$

We consider the mean curvature profile $C_{\tilde{S}}(\tilde{s})=(f(\tilde{s}), g(\tilde{s}))$ associated with the surface $\tilde{S}:\left(\tilde{x}(\tilde{s}) e^{i t}, \tilde{z}(\tilde{s})\right)$. We obtain from (4-3) and (4-4) that

$$
\frac{\tilde{z}_{\tilde{s}}}{\tilde{x}}=\frac{f g_{\tilde{s}}-f_{\tilde{s}} g}{f^{2}+g^{2}}=-\frac{d}{d \tilde{s}}(\arctan (f / g)) .
$$

By using (5-4), a useful formula is obtained by inverting Equations (4-3) and (4-4):

$$
g-i f=(\operatorname{sgn} \tilde{x}) \tilde{x} \exp \left(i \int_{0}^{\tilde{z}} \frac{d \tilde{z}}{\tilde{x}}\right) .
$$

From (4-1) and (5-4), we obtain $\tilde{k}_{2}=-d(\arctan (f / g)) / d \tilde{s}$. Also, it follows from (4-1) and the definition of $\vartheta$ above that $\vartheta_{\tilde{s}}=\tilde{k}_{1}$, and therefore

$$
\theta=\int_{0}^{\tilde{s}} 2 \tilde{H} d \tilde{s}=\int_{0}^{\tilde{s}}\left(\tilde{k}_{1}+\tilde{k}_{2}\right) d \tilde{s}=\vartheta-\arctan (f / g)+c_{3},
$$


where $c_{3}$ is a constant. This gives $\vartheta-\theta=\arctan (f / g)-c_{3}$, which, in (5-3), gives

$$
\begin{aligned}
\Gamma^{*} & =-\frac{\operatorname{sgn} a}{|a|} \sqrt{f^{2}+g^{2}} \cdot\left[\left(\sin \left(\arctan (f / g)-c_{3}\right), \cos \left(\arctan (f / g)-c_{3}\right)\right]\right. \\
& =-\frac{\operatorname{sgn} a}{|a|} \sqrt{f^{2}+g^{2}}\left[\left(\begin{array}{rr}
\cos c_{3} & -\sin c_{3} \\
\sin c_{3} & \cos c_{3}
\end{array}\right)\left(\begin{array}{r}
\sin (\arctan (f / g)) \\
\cos (\arctan (f / g))
\end{array}\right)\right]^{t} \\
& =-\frac{\operatorname{sgn} a}{|a|}\left[\left(\begin{array}{rr}
\cos c_{4} & -\sin c_{4} \\
\sin c_{4} & \cos c_{4}
\end{array}\right)\left(\begin{array}{c}
f \\
g
\end{array}\right)\right]^{t},
\end{aligned}
$$

where $c_{4}=c_{3}$ or $c_{3}+\pi$. Therefore, the curve $\Gamma^{*}$ is a homothety of a rotation of the curve $C_{\tilde{S}}$.

The above discussion and Remark 3.2, and Lemma 5.1 prove the following:

Theorem 5.1. Let $S$ be a smooth surface of revolution generated by the curve

$$
\Omega(s)=(x(s), z(s)) \quad \text { with } \quad x>0 \quad \text { for } \quad l_{1} \leq s \leq l_{2},
$$

(where $l_{1} \leq 0<l_{2}$ ) which is represented by the arc length $s$ and has the $z$-axis as its rotation axis. Assume that the surface $S$ restricted to $\Omega$ has at most isolated umbilics. Assume also that $z^{\prime}$ has at most isolated zeros. Also we assume that $z^{\prime} \neq 0$ at any umbilic of $S$. Regard $\Gamma$ as a curve in the projective plane if $z^{\prime}$ has isolated zeros. Then the rolling curve $\Gamma$ of $\Omega$ is a piecewise $C^{1}$ curve which is smooth away from the umbilics of $S$. Define $\tilde{\Omega}=(\tilde{x}, \tilde{z})$ by $(5-2)$. Let $C_{\tilde{S}}$ be the mean curvature profile associated with the surface $\tilde{S}$ of revolution generated by $\tilde{\Omega}$. Then, the rolling curve $\Gamma$ of $\Omega$ satisfies, up to homothety, $\Gamma=C_{\tilde{S}}^{*}$, that is, $\Gamma$ is (up to homothety) dual to $C_{\tilde{S}}=(f, g)$ with respect to the origin of the coordinate plane. Moreover, the pole of the rolling construction is the origin in this plane. Finally, $\Gamma$ is a closed curve if, for some integer $n$,

$$
\frac{x\left(l_{1}\right)}{z^{\prime}\left(l_{1}\right)}=\frac{x\left(l_{2}\right)}{z^{\prime}\left(l_{2}\right)} \quad \text { and } \quad \int_{l_{1}}^{l_{2}}\left(k_{2}-k_{1}\right) d s=2 n \pi .
$$

Remark 5.1. Each zero of $\tilde{H}$ corresponds to an umbilic point of $S$, and vice versa.

Remark 5.2. Kenmotsu [2003] studied periodic surfaces of revolution with a prescribed mean curvature function. He divided the periodic surfaces of revolution into two classes. One class, which appears there as [Theorem 2], has a mean curvature function for a one parameter family of periodic surfaces of revolution. In the class appearing in [Theorem 3], each mean curvature function has only an isolated periodic surface of revolution. It can be shown that the surfaces appearing in [Theorem 2] are exactly those periodic surfaces whose rolling curves are closed, while those appearing in [Theorem 3] are exactly those periodic surfaces whose rolling curves are not closed. 


\section{Rolling curve of the Wulff shape}

We assume that the Wulff shape $W$ is a smooth convex surface that is rotationally symmetric with respect to the vertical axis (z-axis). We consider the rolling curve $\Gamma: r=r(\theta)$ of the generating curve $\Omega_{W}$ of $W$, and we will obtain a sufficient condition such that $\Gamma$ is smooth.

As usual, we adopt the notations that appeared in (2-1) and (2-2) for the generating curve $\Omega_{W}$ of $W$. We denote by " " " the derivative with respect to $\sigma$. We denote by $\kappa$ the curvature of $\Omega_{W}$ with respect to the inward pointing normal, that is $\kappa:=-u^{\prime \prime} v^{\prime}+u^{\prime} v^{\prime \prime}$.

Theorem 6.1. If either (i) $\kappa^{\prime}<0$ in $-L_{1}<\sigma<0$ and $\kappa^{\prime}>0$ in $0<\sigma<L_{2}$, or (ii) $\kappa^{\prime}>0$ in $-L_{1}<\sigma<0$ and $\kappa^{\prime}<0$ in $0<\sigma<L_{2}$, then the rolling curve $\Gamma$ of the interior $(u(\sigma), v(\sigma))$ for $-L_{1}<\sigma<L_{2}$ of $\Omega_{W}$ is a smooth arc. In (i), $r^{\prime}>0$ in $-L_{1}<\sigma<0$ and $r^{\prime}<0$ in $0<\sigma<L_{2}$. In (ii), $r^{\prime}<0$ in $-L_{1}<\sigma<0$ and $r^{\prime}>0$ in $0<\sigma<L_{2}$.

To prove Theorem 6.1, we prepare a lemma. As usual, we write

$$
\mu_{1}=\kappa=-u^{\prime \prime} v^{\prime}+u^{\prime} v^{\prime \prime} \text { and } \mu_{2}=u^{-1} v_{\sigma} .
$$

Lemma 6.1. If $\kappa^{\prime}<0$ in $-L_{1}<\sigma<0$ and $\kappa^{\prime}>0$ in $0<\sigma<L_{2}$, then $\mu_{1}<\mu_{2}$ in $-L_{1}<\sigma<L_{2}$. If $\kappa^{\prime}>0$ in $-L_{1}<\sigma<0$ and $\kappa^{\prime}<0$ in $0<\sigma<L_{2}$, then $\mu_{1}>\mu_{2}$ in $-L_{1}<\sigma<L_{2}$. Therefore, in both cases, the Wulff shape $W$ has umbilic points only at $(0,0, \min v)$ and $(0,0, \max v)$.

Proof. It is clear that

$$
\mu_{1}=\mu_{2} \quad \text { at } \sigma=-L_{1}, L_{2}
$$

We compute

$$
\mu_{2}^{\prime}=\frac{v^{\prime \prime} u-v^{\prime} u^{\prime}}{u^{2}}=\frac{\kappa u^{\prime} u-v^{\prime} u^{\prime}}{u^{2}}=\frac{u^{\prime}}{u}\left(\mu_{1}-\mu_{2}\right) .
$$

First assume that $\kappa^{\prime}<0$ in $-L_{1}<\sigma<0$ and $\kappa^{\prime}>0$ in $0<\sigma<L_{2}$. Set $f(\sigma):=u^{2}(\sigma)+v^{2}(\sigma)$. We claim that $f(\sigma)$ is a strictly decreasing function of $\sigma$ in $-L_{1} \leq \sigma \leq 0$ and a strictly increasing function in $0 \leq \sigma \leq L_{2}$. In fact, this is proved by a similar way to the proof of [Koiso and Palmer 2007b, Lemma 3.2]. This implies that $\mu_{1}<\mu_{2}$ at $\sigma=0$. Assume $\mu_{1}\left(\sigma_{0}\right)-\mu_{2}\left(\sigma_{0}\right) \geq 0$ for some point $\sigma_{0} \in\left(0, L_{2}\right)$. Then, because of $(6-1)$, there exists some $\sigma_{1} \in\left(0, L_{2}\right)$ such that

$$
\mu_{1}\left(\sigma_{1}\right)-\mu_{2}\left(\sigma_{1}\right) \geq 0 \quad \text { and } \quad \mu_{1}^{\prime}\left(\sigma_{1}\right)-\mu_{2}^{\prime}\left(\sigma_{1}\right) \leq 0 .
$$

On the other hand, (6-2) with the first inequality of (6-3) implies that $\mu_{2}^{\prime}\left(\sigma_{1}\right) \leq 0$. This with the second inequality of (6-3) implies that $\mu_{1}^{\prime}\left(\sigma_{1}\right) \leq \mu_{2}^{\prime}\left(\sigma_{1}\right) \leq 0$. This contradicts the assumption that $\mu_{1}^{\prime}=\kappa^{\prime}>0$ in $0<\sigma<L_{2}$. Therefore, $\mu_{1}<\mu_{2}$ in 
$0 \leq \sigma<L_{2}$. Similarly we can prove that $\mu_{1}<\mu_{2}$ in $-L_{1} \leq \sigma<0$. The proof for the second case is similar.

Proof of Theorem 6.1. Lemma 6.1 combined with Lemma 3.1 implies the first half of Theorem 6.1 .

We will now prove the second half. We will prove the result only for the first case, because the proof of the second case is similar. We know from (3-1) that $r=u / v^{\prime}$. Hence, we have

$$
r^{\prime}=\frac{-u^{\prime}}{\left(v^{\prime}\right)^{2}}\left(\kappa u-v^{\prime}\right)
$$

Set $g:=\kappa u-v^{\prime}$. Then, $g\left(-L_{1}\right)=g\left(L_{2}\right)=0$ and $g^{\prime}=\kappa^{\prime} u+\kappa u^{\prime}-v^{\prime \prime}=\kappa^{\prime} u$. Hence, in the first case, $g<0$ in $-L_{1}<\sigma<L_{2}$. This with (6-4) implies the result.

Figure 5 shows the rolling curve for the Wulff shape having profile curve $u^{4}+$ $v^{4}=1$. Although the Wulff shape $W$ is smooth, the rolling curve has cusps at points which correspond to umbilics of $W$. The rolling construction continues to work in this case as long as the curve is rolled as described in Section 3.

\section{Applications to anisotropic Delaunay surfaces}

Here we will apply the results of the previous sections when $\Omega$ is an anisotropic Delaunay curve.

We assume that the Wulff shape $W$ is rotationally symmetric with respect to the vertical axis. This means that, from (2-15), there exists a positive function $\mu_{2}$ of one variable such that the surface $S:\left(x(s) e^{i t}, z(s)\right)$ is an anisotropic Delaunay surface if and only if $S$ satisfies

$$
2 \mu_{2}^{-1}\left(-x^{\prime}\right) z^{\prime} x+\Lambda x^{2}=c
$$

for some constants $\Lambda \leq 0$ and $c \in \mathbb{R}$. Equation (7-1) determines the surface uniquely up to translation along $z$ direction.

As usual, we adopt the notations that appeared in (2-1) and (2-2) for the generating curve $\Omega_{W}$ of $W$.

First we apply the "dual curve method" constructed in Section 3-Section 5 to anisotropic Delaunay curves $\Omega$. Denote by $S$ the surface of revolution obtained from the curve $\Omega$ by rotating it around $z$-axis. Denote by $\tilde{S}$ the surface of revolution obtained from the curve $(\tilde{x}(s), \tilde{z}(s))$ by rotating it around $z$-axis, where $\tilde{x}$ and $\tilde{z}$ are defined as in (3-3). Then, it is clear that, if $S$ is a horizontal plane, then $\tilde{S}$ is also a horizontal plane, and, if $S$ is a vertical cylinder, then $\tilde{S}$ is also a vertical cylinder.

Lemma 7.1. If $S$ is the Wulff shape, then $\tilde{S}$ is an anisotropic catenoid with flux parameter $c=-2|a|$.

Proof. In this case $\Lambda=-2$. Now it is easy to see that $2 \mu_{2}^{-1} \tilde{z}_{\tilde{s}} \tilde{x}=-2|a|$. 
Lemma 7.2. Assume that $S$ is an anisotropic catenoid with flux parameter $c$. Then $\tilde{S}$ is homothetic to the Wulff shape W. In fact, $\tilde{S}$ is $2|a / c|$ times $W$.

Proof. It is easy to see that $2 \mu_{2}^{-1} \tilde{z}_{\tilde{s}} \tilde{x}+(c /|a|) \tilde{x}^{2}=0$.

Before we study the isothermic duals of an anisotropic unduloid and nodoid, we give a lemma.

Lemma 7.3. $\tilde{z}=\int \tilde{x}_{u} d v$.

Proof. We compute, by using (2-12) and (3-3),

$$
\tilde{z}=\int d \tilde{z}=\int \frac{d \tilde{z}}{d s} \frac{d s}{d z} d z=-a \int \frac{z^{\prime}}{x^{2}}\left(z^{\prime}\right)^{-1} x_{u} d v=-\int \frac{a}{x^{2}} x_{u} d v=\int \tilde{x}_{u} d v .
$$

Lemma 7.4. Assume that $S$ is an anisotropic unduloid $U$, that is, $S$ satisfies (7-1) for some constants $\Lambda<0$ and $c>0$. Set

$$
\tilde{x}(s):=\frac{c}{|\Lambda| x(s)} \quad \text { and } \quad \tilde{z}(s):=-\frac{c}{|\Lambda|} \int_{0}^{s} \frac{z^{\prime}}{x^{2}} d s .
$$

Then, $\tilde{S}$ is an anisotropic unduloid $\tilde{U}$ which is a translation of $\mathcal{U}$ along the $z$ direction, that is, an anisotropic unduloid is isothermic self-dual. A positively curved part of $U$ corresponds to a negatively curved part of $U$; the same holds with "negatively" and "positively" interchanged.

Proof. By using (3-6) and (7-1), we obtain $-2 \mu_{2}^{-1}\left(\tilde{x}_{\tilde{s}}\right) \tilde{x} \tilde{z}_{\tilde{s}}+\Lambda \tilde{x}^{2}=c$. Changing the parameter so that $\tau:=-\tilde{s}$, we obtain

$$
2 \mu_{2}^{-1}\left(-\tilde{x}_{\tau}\right) \tilde{x} \tilde{z}_{\tau}+\Lambda \tilde{x}^{2}=c .
$$

Equation (7-3) means that $(\tilde{x}(\tau), \tilde{z}(\tau))$ generates the same anisotropic unduloid as $(x(s), z(s))$. Therefore, $(\tilde{x}(\tilde{s}), \tilde{z}(\tilde{s}))$ generates the same anisotropic unduloid as $(x(s), z(s))$.

If $S$ is a positively curved part of the anisotropic unduloid $U$, then $x=(u+$ $\left.\sqrt{u^{2}+\Lambda c}\right) /(-\Lambda)$. We obtain using (7-2) that $\tilde{x}=\left(u-\sqrt{u^{2}+\Lambda c}\right) /(-\Lambda)$. This with Lemma 7.3 implies that $\tilde{S}$ is a negatively curved part of $U$.

On the other hand, the same reasoning holds with "negatively" and "positively" interchanged and the sign of $\sqrt{u^{2}+\Lambda c}$ reversed.

Lemma 7.5. Assume that $S$ is an anisotropic nodoid $\mathcal{N}$, that is, $S$ satisfies (7-1) for some constants $\Lambda<0$ and $c<0$. Set

$$
\tilde{x}(s):=-\frac{c}{\Lambda x(s)} \quad \text { and } \quad \tilde{z}(s):=\frac{c}{\Lambda} \int_{0}^{s} \frac{z^{\prime}}{x^{2}} d s .
$$

Then, $\tilde{S}$ is an anisotropic nodoid $\tilde{\mathcal{N}}$ which is a translation of $\mathcal{N}$ along z direction, that is, an anisotropic nodoid is isothermic self-dual. A positively curved part of 
$\mathcal{N}$ corresponds to a negatively curved part of $\mathcal{N}$; the same holds with "negatively" and "positively" interchanged.

Proof. By using (3-6) and (7-1), we obtain

$$
2 \mu_{2}^{-1}\left(-\tilde{x}_{\tilde{s}}\right) \tilde{x} \tilde{z}_{\tilde{s}}+\Lambda \tilde{x}^{2}=c .
$$

The Equation (7-5) means that $(\tilde{x}(\tilde{s}), \tilde{z}(\tilde{s}))$ generates the same anisotropic nodoid as $(x(s), z(s))$.

If $(x(s), z(s))$ generates a positively curved part of the anisotropic nodoid, then $x=\left(u+\sqrt{u^{2}+\Lambda c}\right) /(-\Lambda)$ for $u>0$. We obtain, by using (7-4), $\tilde{x}=-(u+$ $\left.\sqrt{u^{2}+\Lambda c}\right) /(-\Lambda)$ for $u>0$. This with Lemma 7.3 implies that $(\tilde{x}, \tilde{z})$ generates a negatively curved part of $\mathcal{N}$.

On the other hand, the same reasoning holds with "positively" replaced by "negatively" and $u<0$ replaced by $u>0$.

Applying Theorem 5.1 to an anisotropic catenoid using Lemmas 7.2 and 4.1, we obtain the following:

Theorem 7.1. Let $\Omega_{W}(\sigma)$ for $-L_{1} \leq \sigma \leq L_{2}$ be the generating curve of $W$ as usual. Assume that the mean curvature $H_{W}(\sigma)$ of $W$ regarded as a function of $\sigma$ has only isolated zeros. Let $C_{W}(\sigma)$ denote the mean curvature profile associated with $W$. Let $\Omega$ be the generating curve of an anisotropic catenoid with flux parameter $c$ normalized by $c=2$ and denote its rolling curve with $z$-axis as base by $\Gamma$. Then the curve $\Gamma$ is obtained as the dual curve of $C_{W}$, that is $\Gamma=\left(C_{W}\right)^{*}$. Here, the pole of the rolling construction is the origin in the coordinate plane where $C_{W}$ is expressed as $C_{W}(\sigma)=(f(\sigma), g(\sigma))$, which satisfies (4-3) and (4-4) for $(x(s), z(s))=(u(\sigma), v(\sigma))$. In addition, $\Gamma$ is a piecewise $C^{1}$ curve which is smooth away from those points that correspond to the zeros of $H_{W}(\sigma)$ under the anisotropic Gauss map.

Figures 6 and 7 show an anisotropic catenary, an anisotropic parabola (the rolling curve) and the mean curvature profile for the functional whose Wulff shape is generated by a curve

$$
|u|^{p}+|v|^{p}=1
$$

with $p=4$.

Applying Theorem 5.1 to an anisotropic unduloid and nodoid using Lemmas 7.4, 7.5 and 4.1, we obtain the following:

Theorem 7.2. Let $\Omega:(x(s), z(s))$ for $-l \leq s \leq l$ be one period (from a bulge to the next bulge) of the generating curve of an anisotropic unduloid $\mathcal{U}$. Denote by $H_{\Omega}(s)$ the restriction of the mean curvature of $u$ to $\Omega(s)$. We assume that $H_{\Omega}(s)$ vanishes only at isolated points. Define a curve $C_{\Omega}(s)=(f(s), g(s))$ using (4-2) 

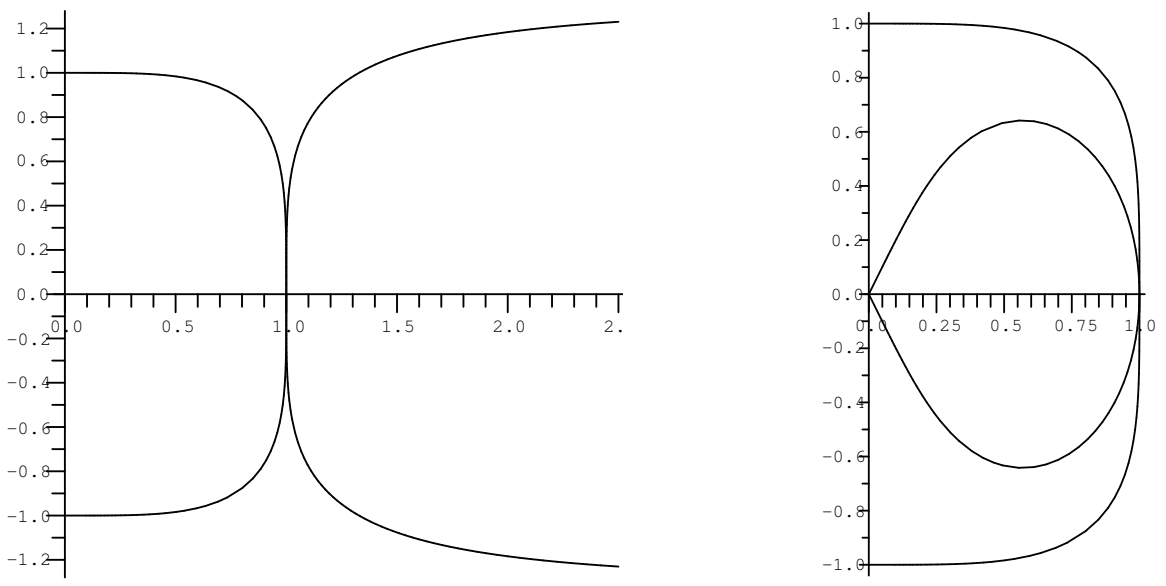

Figure 6. Left: the profile curves of a Wulff shape defined by $u^{4}+v^{4}=1$ (left) and an anisotropic catenoid. These surfaces are isothermic dual to each other. Right: the profile curve of a Wulff shape $W$ defined by $u^{4}+v^{4}=1$ (outer curve) and the mean curvature profile associated with $W$.
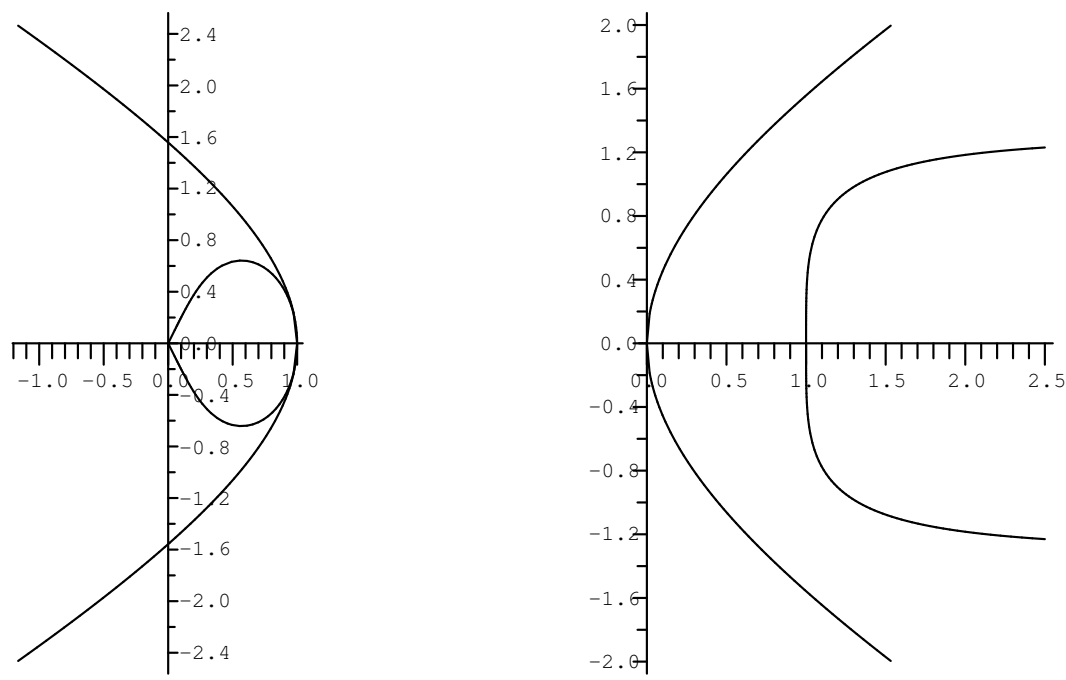

Figure 7. Left: the same mean curvature profile as the one at right in Figure 6 and its dual curve. The dual curve is an anisotropic parabola: the rolling curve of an anisotropic catenary in Equation (2-10) with the origin as the pole. Right: anisotropic catenary (right) and anisotropic parabola. The base of the rolling construction is the vertical axis. 
with $c_{1}=0$ and $c_{2}=-B$, where $B$ is the radius of the bulge. Then, $C_{\Omega}$ is the mean curvature profile associated with $U$. Also, the dual curve $C_{\Omega}^{*}$ of $C_{\Omega}$, with respect to the origin, is a homothety of the rolling curve of one period (from a neck to the next neck) of the generating curve of U with z-axis as base. Here, the pole of the rolling construction is the origin in the coordinate plane where $C_{\Omega}$ is expressed as $C_{\Omega}(s)=(f(s), g(s))$. If we normalize $u$ so that $|c / \Lambda|=1$, then $C_{\Omega}^{*}$ is the rolling curve of one period (from one neck to the next) of the generating curve of $U$ (up to rigid motion). The entire theorem also holds with $u$ replaced by an anisotropic nodoid $\mathcal{N}$.

Figure 8 shows the rolling curves for anisotropic unduloids having Wulff shape of the form (7-6). Figure 9 shows the rolling curve of an anisotropic nodoid having Wulff shape of the form (7-6). Figure 10 demonstrates the rolling construction for an anisotropic unduloid.

In Theorem 7.2 we assumed that the mean curvature of the anisotropic Delaunay curve has at most isolated zeros. Here we give sufficient conditions for the Wulff shape so that this assumption is satisfied.

Lemma 7.6. Assume that the curvature $\kappa$ of the generating curve $\Omega_{W}$ of $W$ satisfies (i) in Theorem 6.1. Then the mean curvature $H$ of an anisotropic unduloid with respect to the outward pointing normal is everywhere negative.

Proof. In Lemma 3.1, we found that the curvature $\kappa_{\Gamma}$ of the rolling curve $\Gamma$ of a general curve $(x(s), z(s))$ is

$$
\kappa_{\Gamma}=\frac{-\left(z^{\prime}\right)^{3}}{x\left|\kappa x-z^{\prime}\right|}=\frac{-\left(z^{\prime}\right)^{3}}{2|a \tilde{H}|} .
$$
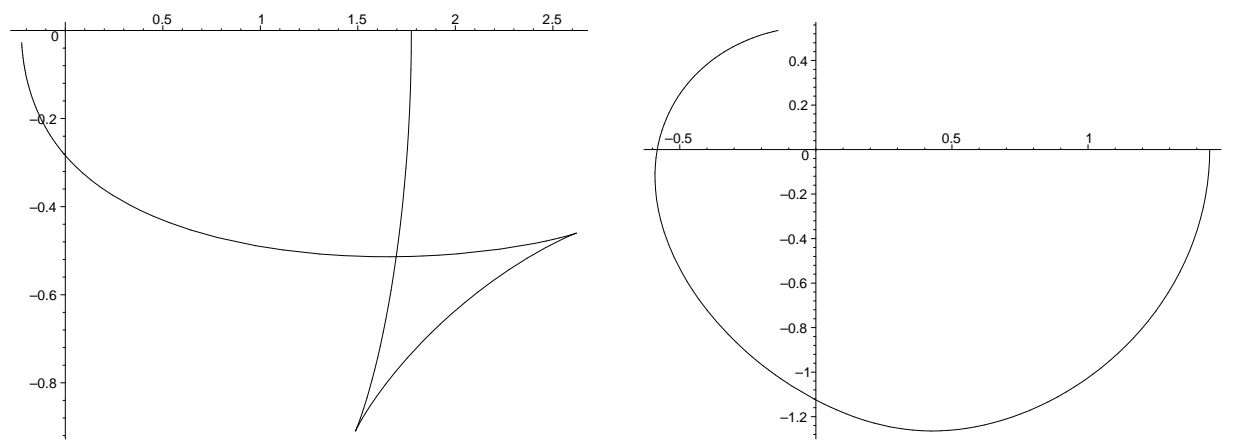

Figure 8. Rolling curves of half periods of an anisotropic undulary with the pole at the origin. Here $p=4$ and $\Lambda=-1$. Left: $c=0.4$. Right: $c=0.8$. 

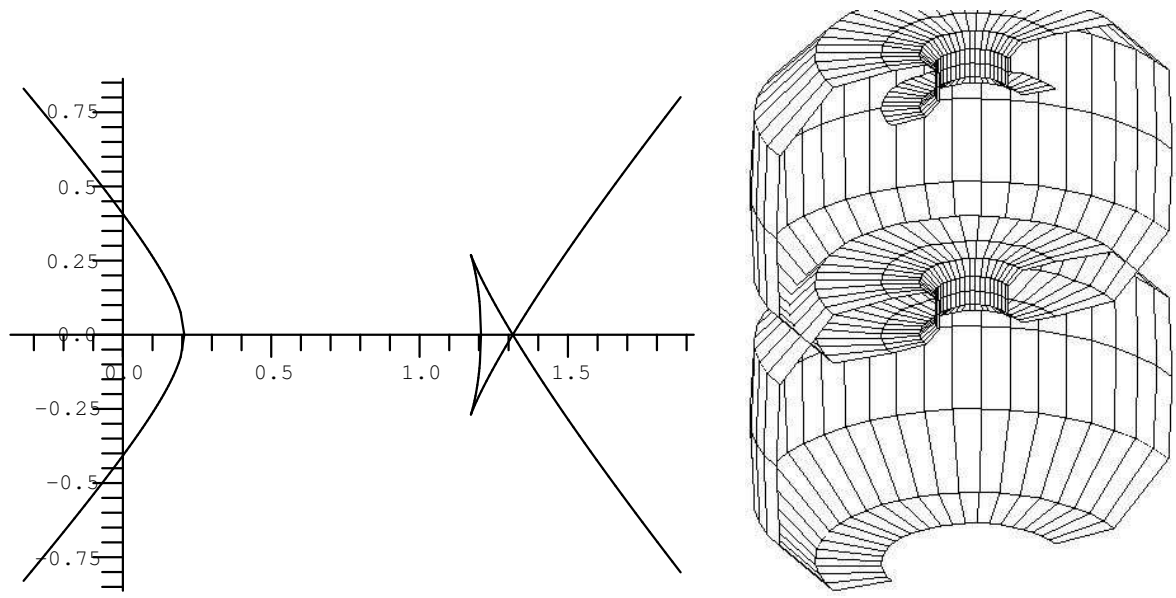

Figure 9. Left: rolling curve of an anisotropic nodary with $p=4$, $\Lambda=-2$, and $c=-0.5$. The pole is the origin. Right: a part of the corresponding anisotropic nodoid.
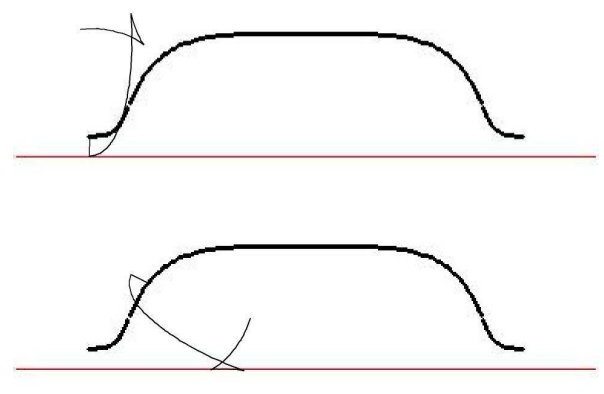

Figure 10. This series of pictures demonstrates the rolling construction for an anisotropic unduloid. The generating curve of the Wulff shape is given by $u^{4}+v^{4}=1, c=0.5$ and $\Lambda=-1$.

Now let $(x(s), z(s))$ be an anisotropic undulary. Then,

$$
x=\frac{u \pm \sqrt{u^{2}+\Lambda c}}{-\Lambda} \text { for } \Lambda>0 \text { and } c>0 .
$$

Set $k_{1}:=-x^{\prime} z^{\prime \prime}+x^{\prime \prime} z^{\prime}$ and $k_{2}:=-z^{\prime} / x$. We compute

$$
k_{1}=-\mu_{1} \sigma_{s}, \quad x \sigma_{s}= \pm \sqrt{u^{2}+\Lambda c}, \quad z^{\prime}=u \mu_{2}, \quad k_{2}=-\mu_{2} \frac{u}{x},
$$

where $\mu_{1}=\kappa=u_{\sigma} v_{\sigma \sigma}-u_{\sigma \sigma} v_{\sigma}$ and $\mu_{2}=v_{\sigma} / u$. Hence, we obtain

$$
H:=\left(k_{1}+k_{2}\right) / 2=-\frac{1}{2 x}\left( \pm \mu_{1} \sqrt{u^{2}+\Lambda c}+\mu_{2} u\right) .
$$


Now, by the assumption on $\kappa$ and Lemma $6.1,0<\mu_{1} \leq \mu_{2}$ for $-L_{1}<\sigma<L_{2}$. This, combined with (7-7), implies $H<0$.

Lemma 7.7. The mean curvature $H$ of an anisotropic nodoid with respect to the outward pointing normal is everywhere negative if

$$
\Lambda c>\max \left\{\frac{\mu_{2}^{2}-\mu_{1}^{2}}{\mu_{1}^{2}} u^{2}\right\} .
$$

If the curvature $\kappa$ of the generating curve $\Omega_{W}$ of $W$ satisfies (ii) in Theorem 6.1, then $H<0$.

Proof. Let $(x(s), z(s))$ be an anisotropic nodary. Then,

$$
x=\frac{u+\sqrt{u^{2}+\Lambda c}}{-\Lambda} \text { for } \Lambda<0 \text { and } c<0 .
$$

As in the proof of Lemma 7.6, we obtain

$$
H=-\frac{1}{2 x}\left(\mu_{1} \sqrt{u^{2}+\Lambda c}+\mu_{2} u\right) .
$$

Therefore, if $u \geq 0$, then $H<0$.

Suppose $u<0$. Then, $\mu_{1} \sqrt{u^{2}+\Lambda c}+\mu_{2} u>0$ if and only if

$$
\Lambda c>\frac{\mu_{2}^{2}-\mu_{1}^{2}}{\mu_{1}^{2}} u^{2} .
$$

This implies the first half of the lemma.

Now, assume (ii) in Theorem 6.1. Then by Lemma 6.1, $\mu_{1} \geq \mu_{2}>0$ for $-L_{1}<$ $\sigma<L_{2}$. Hence (7-8) is satisfied.

We will now briefly discuss how these theorems apply to the classical Delaunay surfaces. Let $\left(x(s) e^{i t}, z(s)\right)$ be a (CMC) catenoid. We make the normalization $c=2$. We take $a=1$. Then $(\tilde{x}, \tilde{z})$ is a half circle with radius 1 and center in the $z$-axis, and $\tilde{H} \equiv-1$. We may assume $\tilde{z}_{\tilde{s}}(0)=1$. Then, by Lemma $4.1, c_{1}=0$ and $c_{2}=-\tilde{x}(0)=-1$. Hence $(f, g)$ is a circle with radius $1 / 2$ and center at $(0,1 / 2)$. Therefore, the rolling curve $\Gamma$ (the dual curve of $(f, g)$ ) is a parabola.

We will apply Theorem 7.2 to the classical unduloid. Let $\left(x(s) e^{i t}, z(s)\right)$ be one period (from a bulge to the next bulge) of an unduloid with mean curvature $H=\Lambda / 2<0$. We take $a=|c / \Lambda|=|c /(2 H)|$. We normalize $|c / \Lambda|=|c /(2 H)|=1$. Then $(\tilde{x}, \tilde{z})$ gives the same undulary as $(x(s), z(s))$ (up to translation). We may assume $z^{\prime}(0)=1$. Then $B=(1+\sqrt{1-2|H c|}) /|2 H|$. Since the mean curvature 
of $(x, z)$ is a constant $H$,

$$
\begin{aligned}
& f(s)=\int_{0}^{s} \cos (2 H s) d s=\frac{1}{2 H} \sin (2 H s) \\
& g(s)=\int_{0}^{s} \sin (2 H s) d s+B=-\frac{1}{2 H} \cos (2 H s)+\frac{\sqrt{1-2|H c|}}{2|H|} .
\end{aligned}
$$

Thus $(f, g)$ is a circle with radius $1 /|2 H|$ and center at $(0, \sqrt{1-2|H| c} /|2 H|)$. The origin is inside of the circle $(f, g)$. Therefore, the rolling curve $\Gamma$ (the dual curve of $(f, g))$ is an ellipse.

Finally, we apply Theorem 7.2 to the nodoid. Let $\left(x(s) e^{i t}, z(s)\right)$ be one period (from one bulge to the next) of a nodoid with mean curvature $H=\Lambda / 2<0$. We take $a=-|c / \Lambda|=-|c /(2 H)|$. We normalize $|c / \Lambda|=|c /(2 H)|=1$. Then $(\tilde{x}, \tilde{z})$ gives the same nodary as $(x(s), z(s))$ (up to translation). We may assume $z^{\prime}(0)=1$. Then $B=(1+\sqrt{1+2|H c|}) /|2 H|$. Since the mean curvature of $(\tilde{x}, \tilde{z})$ is a constant $H$,

$$
\begin{aligned}
& f(s)=\int_{0}^{s} \cos (2 H s) d s=\frac{1}{2 H} \sin (2 H s) \\
& g(s)=\int_{0}^{s} \sin (2 H s) d s+B=-\frac{1}{2 H} \cos (2 H s)+\frac{\sqrt{1+2|H c|}}{2|H|} .
\end{aligned}
$$

Thus $(f, g)$ is a circle with radius $1 /|2 H|$ and center at $(0, \sqrt{1+2|H| c} /|2 H|)$. The origin is outside of the circle $(f, g)$. Therefore, the rolling curve $\Gamma$ (the dual curve of $(f, g))$ is a hyperbola.

To conclude this section, we give expressions of the rolling curves $\Gamma$ of the anisotropic undulary and nodary in terms of the Wulff shape, which are useful for drawing pictures of $\Gamma$. It is sufficient to give formulas only for the "half" period that corresponds to a part of the "upper half" $(u(\sigma), v(\sigma))$ for $0 \leq \sigma \leq L_{2}$ of $\Omega_{W}$.

Proposition 7.1. Let $S$ be a "half" period of an anisotropic unduloid $U$ with anisotropic mean curvature $\Lambda$ and flux parameter $c$. Parametrize the corresponding half period of the generating curve of the Wulff shape as $u=u(v)$ for $0 \leq v \leq$ $\bar{v}:=\max v$ with $u \geq 0$, and let $v_{1}>0$ be defined by $u\left(v_{1}\right)=\sqrt{-\Lambda c}$. Then the $x$ coordinate of the generating curve $C$ of $S$ can be represented as

$$
x= \begin{cases}\frac{u+\sqrt{u^{2}+\Lambda c}}{-\Lambda} & \text { for } v=0 \text { to } v=v_{1}, \\ \frac{u-\sqrt{u^{2}+\Lambda c}}{-\Lambda} & \text { for } v=v_{1} \text { to } v=0 .\end{cases}
$$


Then, the rolling curve $\Gamma:(r, \theta)$ is given as follows:

$$
r= \begin{cases}\frac{u+\sqrt{u^{2}+\Lambda c}}{-\Lambda} \cdot \sqrt{1+u_{v}^{2}} & \text { for } v=0 \text { to } v=v_{1}, \\ \frac{u-\sqrt{u^{2}+\Lambda c}}{-\Lambda} \cdot \sqrt{1+u_{v}^{2}} & \text { for } v=v_{1} \text { to } v=0 .\end{cases}
$$

$\theta= \begin{cases}\arcsin \left(\frac{\left|u_{v}\right|}{\sqrt{1+u_{v}^{2}}}\right)-\int_{0}^{v} \frac{d v}{\sqrt{u^{2}+\Lambda c}} & \text { for } v=0 \text { to } v=v_{1}, \\ \arcsin \left(\frac{\left|u_{v}\right|}{\sqrt{1+u_{v}^{2}}}\right)+\int_{v_{1}}^{v} \frac{d v}{\sqrt{u^{2}+\Lambda c}}-\int_{0}^{v_{1}} \frac{d v}{\sqrt{u^{2}+\Lambda c}} \text { for } v=v_{1} \text { to } v=0 .\end{cases}$

In particular, if $W$ is symmetric with respect to the horizontal plane, the rolling curve of one period of $U$ is a closed curve if and only if

$$
\int_{0}^{v_{1}} \frac{d v}{\sqrt{u^{2}+\Lambda c}}=\frac{\pi}{2} .
$$

Proof. From (3-1), we have $r=x / z^{\prime}=x / v_{\sigma}$. On the interval in question, we have $v_{\sigma}^{2}=\left(1+u_{v}^{2}\right)^{-1}$, from which we obtain (7-9).

Recall (2-16) and (3-2). We compute

$$
\begin{aligned}
\theta & =-\int\left(\frac{z^{\prime}}{x}-\kappa\right) d s=-\int\left(\frac{v_{\sigma}}{x}-\left(u_{\sigma} v_{\sigma \sigma}-u_{\sigma \sigma} v_{\sigma}\right) \frac{d \sigma}{d s}\right) d s \\
& =-\int \frac{d v}{x \sigma_{s}}-\int \frac{u_{\sigma \sigma}}{v_{\sigma}} d \sigma=-\int \frac{x_{u}}{x} d v+\arcsin \left(\frac{\left|u_{v}\right|}{\sqrt{1+u_{v}^{2}}}\right)+\text { constant } \\
& =\mp \int \frac{d v}{\sqrt{u^{2}+\Lambda c}}+\arcsin \left(\frac{\left|u_{v}\right|}{\sqrt{1+u_{v}^{2}}}\right)+\text { constant. }
\end{aligned}
$$

In a similar way, and using Lemma 5.1, we have the following.

Proposition 7.2. Suppose $S$ is a "half" period of an anisotropic nodoid $\mathcal{N}$ with anisotropic mean curvature $\Lambda$ and flux parameter $c$. Parametrize the upper half of the generating curve of the Wulff shape as

$$
v=v(u) \geq 0 \quad \text { for }-\bar{u} \leq u \leq \bar{u}:=\max u .
$$

On this interval the $x$ coordinate of the generating curve $C$ of $S$ can be represented as, by setting $\bar{v}:=\max v$,

$$
x=\frac{u+\sqrt{u^{2}+\Lambda c}}{-\Lambda} \quad \text { for }\left\{\begin{aligned}
0 \leq u \leq \bar{u} & \text { as } v=0 \text { to } v=\bar{v}, \\
-\bar{u} \leq u<0 & \text { as } v=\bar{v} \text { to } v=0 .
\end{aligned}\right.
$$

Then, the rolling curve $\Gamma:(r, \theta)$ is

$$
r=\frac{u+\sqrt{u^{2}+\Lambda c}}{-\Lambda} \cdot \sqrt{1+u_{v}^{2}} \quad \text { for }\left\{\begin{aligned}
0 \leq u \leq \bar{u} & \text { as } v=0 \text { to } v=\bar{v} \\
-\bar{u} \leq u<0 & \text { as } v=\bar{v} \text { to } v=0
\end{aligned}\right.
$$




$$
\theta= \begin{cases}\arcsin \left(\frac{\left|u_{v}\right|}{\sqrt{1+u_{v}^{2}}}\right)-\int_{0}^{v} \frac{d v}{\sqrt{u^{2}+\Lambda c}} & \text { for } v=0 \text { to } v=\bar{v}, \\ 2 \pi-\arcsin \left(\frac{\left|u_{v}\right|}{\sqrt{1+u_{v}^{2}}}\right)-\int_{0}^{v} \frac{d v}{\sqrt{u^{2}+\Lambda c}} & \text { for } v=\bar{v} \text { to } v=0,\end{cases}
$$

where $\arcsin \left(\left|u_{v}\right| / \sqrt{1+u_{v}^{2}}\right)$ takes values in the closed interval $[0, \pi / 2]$. In particular, the total variation of $\theta$ for one period of $\mathcal{N}$ is $2 \pi$, and therefore its rolling curve is a closed curve (in the projective plane).

\section{Characterization of anisotropic Delaunay curves}

What surfaces of revolution arise as anisotropic Delaunay surfaces? Here, using isothermic duality, we characterize the surfaces which can arise as anisotropic unduloids and nodoids without making explicit reference to the functional.

Let $\Omega(s)=(x(s), z(s))$ with $x(s)>0$ be a smooth curve with arc length $s$ which is periodic with period $L$ in the following sense: $x$ and $z$ satisfy $x(s+L)=x(s)$ and $z(s+L)-z(s)=z(L)-z(0)$ for all $s \in \mathbb{R}$. We assume that one period of $\Omega$ contains a unique local maximum and a unique local minimum of $x$, which will be called a bulge and a neck of $\Omega$, respectively. We also assume that $\Omega$ satisfies either of these conditions:

(I) There is only one inflection point between a bulge and the next neck, and there is no zero of $z^{\prime}$.

(II) There is no inflection point, and there is only one zero of $z^{\prime}$ between a bulge and the next neck.

We may assume that $s=0$ corresponds to a bulge, $s=-l_{1}, l_{2}$ with $l_{1}, l_{2}>0$ correspond to the next necks, and $z(0)=0$. We denote by $-s_{I}$ and $s_{J}$ with $-l_{1}<$ $-s_{I}<0<s_{J}<l_{2}$ the unique point in (I) or (II) above.

Define a curve $\tilde{\Omega}(s):=(\tilde{x}(s), \tilde{z}(s))$,

$$
\tilde{x}(s):=\frac{a}{x(s)} \quad \text { and } \quad \tilde{z}(s):=-a \int_{0}^{s} \frac{z^{\prime}}{x^{2}} d s,
$$

as before. Here, $a$ is a positive constant if $\Omega$ satisfies (I), and $a$ is a negative constant if $\Omega$ satisfies (II).

Theorem 8.1. If $\Omega(\mathbb{R})=\tilde{\Omega}(\mathbb{R})$ up to vertical translation and reflection with respect to the vertical axis (z-axis), then $\Omega$ is an anisotropic undulary or nodary for some anisotropic surface energy $\mathscr{F}=\int F\left(v_{3}\right) d \Sigma$ with rotationally symmetric energy integrand.

Conversely, if $\Omega$ is an anisotropic undulary or nodary for an anisotropic surface energy $\mathscr{F}=\int F\left(v_{3}\right) d \Sigma$ with rotationally symmetric energy integrand, then $\Omega$ is periodic, either $(I)$ or $(I I)$ holds, and $\Omega(\mathbb{R})=\tilde{\Omega}(\mathbb{R})$ for some constant $a \neq 0$ up to vertical translation. 
Proof. We need to prove only the first half. We know from (3-6) that

$$
\left(\tilde{x}_{\tilde{s}}, \tilde{z}_{\tilde{s}}\right)= \begin{cases}\left(-x^{\prime},-z^{\prime}\right) & \text { if } a>0 \\ \left(x^{\prime}, z^{\prime}\right) & \text { if } a<0\end{cases}
$$

First we assume that (I) is satisfied. We may assume that $z^{\prime}(s)>0$ for all $s$. The range $z^{\prime}\left(\left[-l_{1}, 0\right]\right)$ is a closed interval $\left[\beta_{1}, 1\right]$, and $z^{\prime}\left(\left[0, l_{2}\right]\right)$ is a closed interval $\left[\beta_{2}, 1\right]$ for some $\beta_{1}, \beta_{2} \in(0,1)$. Each point satisfying $x^{\prime}>0$ and $z^{\prime}=\beta_{1}$ corresponds to the inflection point $s=-s_{I}$; each point satisfying $x^{\prime}<0$ and $z^{\prime}=\beta_{2}$ corresponds to the inflection point $s=s_{J}$. We may assume that $s=0$ corresponds to $\tilde{s}=0$. Because of (8-1) with $a>0$, the range $\tilde{z}_{\tilde{s}}\left(\left[-l_{1}, 0\right]\right)$ is a closed interval $\left[-1,-\beta_{1}\right]$, and $\tilde{z}_{\tilde{s}}\left(\left[0, l_{2}\right]\right)$ is a closed interval $\left[-1,-\beta_{2}\right]$.

Then, at $\tilde{s}=l_{2}-s_{J}$,

$$
x\left(s_{J}\right)=\tilde{x}\left(l_{2}-s_{J}\right) \quad \text { and } \quad\left(x^{\prime}\left(s_{J}\right), z^{\prime}\left(s_{J}\right)\right)=\left(-\tilde{x}_{\tilde{s}}\left(l_{2}-s_{J}\right),-\tilde{z}_{\tilde{s}}\left(l_{2}-s_{J}\right)\right) .
$$

Therefore, $a=x^{2}\left(s_{J}\right)$. Similarly, we obtain $a=x^{2}\left(-s_{I}\right)$. Hence we must have $a=x^{2}\left(s_{J}\right)=x^{2}\left(-s_{I}\right)$. Now set $\tilde{\mu}_{2}:=2 z^{\prime} /(x+\tilde{x})$. Then $\tilde{\mu}_{2}$ can be regarded as a function of $\tilde{v}_{3}:=-\tilde{x}_{\tilde{s}}$. For any negative number $\Lambda$, set

$$
\mu_{2}\left(v_{3}\right):=(-\Lambda)^{-1} \tilde{\mu}_{2}\left(-\tilde{v}_{3}\right) \text { and } c:=-a \Lambda .
$$

Then $2 \mu_{2}^{-1} z^{\prime} x+\Lambda x^{2}=c$ for $c>0$. Set

$$
u:=\mu_{2}^{-1} z^{\prime} \quad \text { and } \quad v:=\int_{0}^{z} \frac{z^{\prime}}{x^{\prime}} u^{\prime} d s
$$

Then it is easy to see that $z=\int x_{u} d v$. Denote the arc length of $(u, v)$ by $\sigma$. Then, by elementary computation, we obtain $(d \sigma / d s)^{2}=\left(u^{\prime}\right)^{2} /\left(x^{\prime}\right)^{2}$. Therefore, we obtain $d v / d \sigma=(d v / d s)(d s / d \sigma)=z^{\prime}$. Hence $2 \mu_{2}^{-1} v_{\sigma} u-2 u^{2}=0$, which is the equation for the generating curve of the Wulff shape. Now, $u$ and $v$ can be regarded as functions of $v_{3}$ for $x^{\prime}\left(s_{J}\right) \leq v_{3} \leq x^{\prime}\left(-s_{I}\right)$. We compute

$$
u^{\prime}(s) v^{\prime \prime}(s)-u^{\prime \prime}(s) v^{\prime}(s)=\left(u^{\prime}(s)\right)^{2}\left(x^{\prime}\right)^{-2}\left(x^{\prime} z^{\prime \prime}-x^{\prime \prime} z^{\prime}\right) .
$$

Since the arc $(x(s), z(s))$ for $-s_{I} \leq s \leq s_{J}$ is convex with respect to the inward normal, $u^{\prime}(s) v^{\prime \prime}(s)-u^{\prime \prime}(s) v^{\prime}(s)>0$. Therefore, $(u, v)$ is also convex. $(u, v)$ can be extended to a convex closed curve, which we will denote by $(u, v)$ also. Then, $(x, z)$ is an anisotropic undulary with constant anisotropic mean curvature $\Lambda$ for a Wulff shape generated by $(u, v)$.

If (II) is satisfied, then, in a similar way, we can prove that $(x, z)$ is an anisotropic nodary with constant anisotropic mean curvature $\Lambda$ for a Wulff shape generated by a convex closed curve $(u, v)$. 


\title{
References
}

[Delaunay 1841] C. Delaunay, "Sur la surface de revolution dont la courbure moyenne est constante”, J. Math. Pures et Appl. (1) 6 (1841), 309-320.

[Eisenhart 1960] L. P. Eisenhart, A treatise on the differential geometry of curves and surfaces, Dover, New York, 1960. MR 22 \#5936 Zbl 0090.37803

[Gielis 2003] J. Gielis, "A generic geometric transformation that unifies a large range of natural and abstract shapes", Am. J. of Botany 90:3 (2003), 333-338.

[Guggenheimer 1977] H. W. Guggenheimer, Differential geometry, Dover Publications, New York, 1977. MR 58 \#12737 Zbl 0357.53002

[Hsiang and Yu 1981] W.-Y. Hsiang and W. C. Yu, "A generalization of a theorem of Delaunay", J. Differential Geom. 16:2 (1981), 161-177. MR 83f:53001 Zbl 0504.53044

[Kenmotsu 1980] K. Kenmotsu, "Surfaces of revolution with prescribed mean curvature", Tôhoku Math. J. (2) 32:1 (1980), 147-153. MR 81h:53003 Zbl 0431.53005

[Kenmotsu 2003] K. Kenmotsu, "Surfaces of revolution with periodic mean curvature", Osaka J. Math. 40:3 (2003), 687-696. MR 2004h:53012 Zbl 1041.53005

[Koiso and Palmer 2005] M. Koiso and B. Palmer, "Geometry and stability of surfaces with constant anisotropic mean curvature", Indiana Univ. Math. J. 54:6 (2005), 1817-1852. MR 2006h:58016 Zbl 05014750

[Koiso and Palmer 2006] M. Koiso and B. Palmer, "Stability of anisotropic capillary surfaces between two parallel planes", Calc. Var. Partial Differential Equations 25:3 (2006), 275-298. MR 2006j:58018 Zbl 05014072

[Koiso and Palmer 2007a] M. Koiso and B. Palmer, "Anisotropic capillary surfaces with wetting energy”, Calc. Var. Partial Differential Equations 29:3 (2007), 295-345. MR 2321891 Zbl 05164779

[Koiso and Palmer 2007b] M. Koiso and B. Palmer, "Uniqueness theorems for stable anisotropic capillary surfaces”, SIAM J. Math. Anal. 39:3 (2007), 721-741. MR 2349864

Received February 12, 2007. Revised September 25, 2007.

\author{
MIYUKI KOISO \\ DEPARTMENT OF MATHEMATICS \\ NARA WOMEN'S UNIVERSITY \\ NARA 630-8506 \\ JAPAN \\ koiso@cc.nara-wu.ac.jp \\ BENNETT PALMER \\ DEPARTMENT OF MATHEMATICS \\ IDAHO STATE UNIVERSITY \\ POCATELLO, ID 83209 \\ UNITED STATES \\ palmbenn@isu.edu \\ http://www.isu.edu/ palmbenn
}

